\title{
Matter power spectrum from a Lagrangian-space regularization of perturbation theory
}

\author{
Patrick Valageas \\ Institut de Physique Théorique, \\ CEA, IPhT, F-91191 Gif-sur-Yvette, Cédex, France \\ CNRS, URA 2306, F-91191 Gif-sur-Yvette, Cédex, France \\ Takahiro Nishimichi \\ Kavli Institute for the Physics and Mathematics of the Universe, \\ Todai Institutes for Advanced Study, The University of Tokyo, \\ Kashiwa, Chiba 277-8583, Japan (Kavli IPMU, WPI) \\ Atsushi Taruya \\ Research Center for the Early Universe, School of Science, \\ The University of Tokyo, Bunkyo-ku, Tokyo 113-0033, Japan \\ Kavli Institute for the Physics and Mathematics of the Universe, \\ Todai Institutes for Advanced Study, The University of Tokyo, \\ Kashiwa, Chiba 277-8583, Japan (Kavli IPMU, WPI)
}

(Dated: March 22, 2019)

\begin{abstract}
We present a new approach to computing the matter density power spectrum, from large linear scales to small highly nonlinear scales. Instead of explicitly computing a partial series of highorder diagrams, as in perturbative resummation schemes, we embed the standard perturbation theory within a realistic nonlinear Lagrangian-space ansatz. We also point out that an "adhesionlike" regularization of the shell-crossing regime is more realistic than a "Zel'dovich-like" behavior, where particles freely escape to infinity. This provides a "cosmic web" power spectrum with good small-scale properties that provide a good matching with a halo model on mildly nonlinear scales. We obtain a good agreement with numerical simulations on large scales, better than $3 \%$ for $k \leq$ $1 \mathrm{hpcc}^{-1}$, and on small scales, better than $10 \%$ for $k \leq 10 \mathrm{hpc}^{-1}$, at $z \geq 0.35$, which improves over previous methods.
\end{abstract}

PACS numbers: 98.80.-k, 98.65.Dx

\section{INTRODUCTION}

The description of gravitational clustering can follow two different frameworks: the Eulerian approach, where one studies density and velocity fields on a fixed grid, and the Lagrangian approach, where one studies particle trajectories themselves [1].

The Eulerian approach is more convenient for some practical purposes, because we observe density fields and not trajectories (and it is also better suited to baryonic physics, which involves pressure and temperature fields). However, it has the theoretical disadvantage that one should in principle work with the phase-space distribution function $f(\mathbf{x}, \mathbf{v} ; t)$, which involves seven variables and is very heavy for numerical and analytical computations. Then in practice, most analytical approaches are based on the fluid approximation, which replaces the Vlasov equation with hydrodynamical equations for the density and velocity fields $\rho(\mathbf{x}, t)$ and $\mathbf{v}(\mathbf{x}, t)$, which are much easier to handle. However, this is only exact in the single-stream regime and these equations of motion themselves break down after shell crossing. This makes the matching between the perturbative and nonperturbative regimes rather difficult. More precisely, because the dynamics is not well defined (these equations of motion are not sufficient to determine the evolution after shell crossing), the asymptotic regime at high wave numbers of the power spectrum obtained within this framework is not obvious and not clearly related to physical behaviors.

In contrast, in the Lagrangian approach, the trajectories $\mathbf{x}(\mathbf{q}, t)$, where particles are identified by their initial position $\mathbf{q}$, are always well defined and the behavior after shell crossing is (at least implicitly) defined within each peculiar scheme. This offers the prospect of a more convenient matching to the highly nonlinear regime. Moreover, in contrast to the Eulerian case the Lagrangian approach is not sensitive to the "sweeping effect" associated with almost uniform translations by long wavelengths of the velocity field 2-44] (i.e., this can be automatically removed as a random uniform shift), and it is a more direct probe of the structures of the density field. From an observational point of view, this framework is also more convenient for including redshift-space distortions. However, to compare theoretical predictions with observations, one typically needs to compute again the density fields, and the derivation of the latter from the particle trajectories usually introduces additional approximations and problems.

This latter disadvantage explains why most perturbative schemes that have been recently developed follow the Eulerian framework [5 14]. They provide a good match with numerical simulations on quasilinear scales by using resummation schemes that are exact up to the oneor two-loop order and improve over the standard per- 
turbative approach by including partial resummations of higher-order diagrams. However, it has been difficult so far to obtain a good model up to the highly nonlinear scales (by embedding the perturbative predictions within a phenomenological halo model), as one usually underestimates the power spectrum on transition scales [11, 15]. This discrepancy is even worse for Lagrangian schemes, which usually give rise to a perturbative power spectrum that decays faster than the linear power spectrum at high $k$ [11, 16, 17], whereas the Eulerian schemes can show a variety of behaviors, a similar fast decay, a convergence back to the linear power, or a higher tail.

In this paper, we follow two main motivations:

(1) Develop a Lagrangian-based approach.

(2) Obtain a good matching with the highly nonlinear regime.

Indeed, a Lagrangian-based approach is expected to provide a better starting point for eventually tackling redshift-space distortions and biasing (although we do not address these points here). Moreover, it is not sensitive to the "sweeping effect", so that the modeling can be directly focused on density structures, that is, relative displacements. Next, because our final goal is to obtain a unified description from linear to highly nonlinear scales, it is necessary to pay attention to transition scales. Moreover, the requirement of a good matching to nonlinear scales is likely to shed light on the asymptotic behavior that should be satisfied by good perturbative schemes.

To reach these goals, we advocate a new perspective while drawing on some previous works. Our main new ideas are the following:

(a) Instead of building a perturbative resummation scheme, which explicitly computes a partial series of highorder diagrams, we build a self-consistent ansatz that we next make consistent with standard perturbation theory up to some order (one-loop order in this paper).

(b) Instead of the usual "Zel'dovich-like" behavior in the shell-crossing regime, we impose an "adhesion-like" behavior.

These two strategies are motivated by the wish to obtain a good behavior up to nonlinear scales.

First, most perturbative schemes are not guaranteed to provide at each order self-consistent predictions, whether they involve a sharp truncation at a finite order (as in standard perturbation theory) or include partial resummations of high-order terms. In other words, they do not guarantee that their predictions can be realized by a physical density field (such that the density is real and positive), and one may encounter unphysical behaviors at high $k$ (such as a negative power spectrum). This is not necessarily a problem, if one restricts the focus to the range of validity of the perturbative scheme. However, since we intend to build a unified model that applies up to nonlinear scales, we need to obtain as a building block a perturbative power spectrum that remains well behaved in all regimes. A second reason is that physical self-consistency (at least to some degree) can be expected to ensure good convergence properties or at least a reasonable high- $k$ tail. For instance, this clearly rules out the bad behavior of the standard Eulerian perturbation theory, where higher-order contributions grow increasingly fast on small scales and lead to power spectra that can change sign with the truncation order. Then, we can hope that a better-controlled high- $k$ behavior and a better convergence can in turn improve the accuracy on quasilinear scales.

The method that we develop to achieve this goal is first to build an ansatz for the power spectrum that is always well behaved and next to tune its parameters (in our case, the skewness of the longitudinal displacement) so that its perturbative expansion matches the standard perturbative expansion up to the required order (here up to the second order over $P_{L}$ ).

Second, the "Zel'dovich-like" behavior of usual Lagrangian perturbative schemes, where particles escape to infinity after shell crossing, leads to a fast decay of the power spectrum on nonlinear scales. This is due to the erasing of intermediate-scale structures such as pancakes soon after their formation, because particles do not remain trapped within potential wells. As recalled above, this may be the source of a significant underestimation of the power spectrum on transition scales. The "adhesion model" introduced in Ref.[18], where particles stick together after shell crossing, seems a more realistic approximation. In particular, this provides a good description of the cosmic web. Because it is not easy to implement the exact adhesion model within a model for the power spectrum, we use a simplified ansatz, inspired from Ref. 19], to include some form of "sticking" of particle pairs after shell crossing. This models the formation of pancakes and yields additional power at high $k$, which should be more realistic and more accurate than previous methods.

These ingredients allow us to build a model for the "cosmic web" power spectrum, associated with large and intermediate scale structures (bulk flows, voids, and pancackes). Next, following Refs. [11, 15], we combine these results with a phenomenological halo model to extend our model up to highly nonlinear scales, associated with inner halo regions.

This paper is organized as follows. After recalling the expression of the matter density power spectrum in a Lagrangian-space framework in Sec. III we describe in Sec. III our model for the "cosmic web" power spectrum. This corresponds to both the large-scale perturbative regime and the nonperturbative intermediate-scale regime associated with pancakes. Next, we explain in Sec. IV how we combine this cosmic web power spectrum with a halo model to extend our model to highly nonlinear scales, associated with inner halo regions. Then, we compare our model with numerical simulations in Sec. V. and we conclude in Sec.VI.

The reader who is only interested in our final numerical results may directly go to Sec. V.

In this paper, we mainly focus on a flat $\Lambda \mathrm{CDM}$ cosmology derived from the five-year observation by the WMAP 
satellite [20]. We measure some of the ingredients in our model from five out of 60 realizations of N-body simulations performed in 14, adopting this cosmological model. The numerical data for the power spectrum at large scales $\left(k<1 h \mathrm{Mpc}^{-1}\right)$ is taken from the full 60 simulations in that paper, while we refer to a higherresolution simulation by [11] for smaller scales. Finally, we show how well our model can reproduce the power spectrum from N-body simulations done in two other cosmologies.

\section{MATTER DENSITY POWER SPECTRUM IN A LAGRANGIAN FRAMEWORK}

In a Lagrangian framework one considers the trajectories $\mathbf{x}(\mathbf{q}, t)$ of all particles, of initial Lagrangian coordinates $\mathbf{q}$ and Eulerian coordinates $\mathbf{x}$ at time $t$. In particular, at any given time $t$, this defines a mapping, $\mathbf{q} \mapsto \mathbf{x}$, from Lagrangian to Eulerian space, which fully determines the Eulerian density field $\rho(\mathbf{x})$ through the conservation of matter,

$$
\rho(\mathbf{x}) \mathrm{d} \mathbf{x}=\bar{\rho} \mathrm{d} \mathbf{q},
$$

where $\bar{\rho}$ is the mean comoving matter density of the Universe and we work in comoving coordinates. Then, defining the density contrast as

$$
\delta(\mathbf{x}, t)=\frac{\rho(\mathbf{x}, t)-\bar{\rho}}{\bar{\rho}},
$$

and its Fourier transform as

$$
\tilde{\delta}(\mathbf{k})=\int \frac{\mathrm{d} \mathbf{x}}{(2 \pi)^{3}} e^{-\mathrm{ik} \cdot \mathbf{x}} \delta(\mathbf{x}),
$$

one obtains from Eq.1

$$
\tilde{\delta}(\mathbf{k})=\int \frac{\mathrm{d} \mathbf{q}}{(2 \pi)^{3}}\left(e^{-\mathrm{i} \mathbf{k} \cdot \mathbf{x}(\mathbf{q})}-e^{-\mathrm{i} \mathbf{k} \cdot \mathbf{q}}\right) .
$$

Next, defining the density power spectrum as

$$
\left\langle\tilde{\delta}\left(\mathbf{k}_{1}\right) \tilde{\delta}\left(\mathbf{k}_{2}\right)\right\rangle=\delta_{D}\left(\mathbf{k}_{1}+\mathbf{k}_{2}\right) P\left(k_{1}\right),
$$

we obtain from Eq.(44), using statistical homogeneity [21, 22],

$$
P(k)=\int \frac{\mathrm{d} \Delta \mathbf{q}}{(2 \pi)^{3}}\left\langle e^{\mathrm{i} \mathbf{k} \cdot \Delta \mathbf{x}}-e^{\mathrm{i} \mathbf{k} \cdot \Delta \mathbf{q}}\right\rangle,
$$

where we introduced the Lagrangian-space and Eulerianspace separations $\Delta \mathbf{q}$ and $\Delta \mathbf{x}$,

$$
\Delta \mathbf{q}=\mathbf{q}_{2}-\mathbf{q}_{1}, \quad \Delta \mathbf{x}=\mathbf{x}_{2}-\mathbf{x}_{1},
$$

between two particles $\mathbf{q}_{1}$ and $\mathbf{q}_{2}$. The expression (6) is fully general since it is a simple consequence of the matter conservation [Eq.(10)] and of statistical homogeneity. In particular, it holds for any dynamics, such as the one associated with the Zel'dovich approximation [23], where the mapping $\mathbf{x}(\mathbf{q})$ is given by the linear displacement field.

\section{LARGE-SCALE POWER SPECTRUM (COSMIC WEB)}

Our final goal is to build a unified model for the power spectrum that applies from linear to highly nonlinear scales, which requires at some level a phenomenological model to describe small scales. As described in Sec. IV below, following [11, 15] we use a halo model to combine large-scale perturbative schemes with phenomenological approaches. However, we first focus on our perturbative approach, where we neglect the formation of virialized halos. Thus, we consider in this section the power spectrum associated with large-scale bulk flows, that is, the formation of the cosmic web, disregarding small-scale structures such as inner halo regions. This actually includes two regimes: i) the very large scales where shell crossing can be neglected, and ii) the intermediate scales, associated with pancakes or filaments, where shell crossing comes into play to shape the cosmic web, but where the internal structure of pancakes and filaments can be neglected. The first regime can be described by perturbation theory, and it is considered in Secs. III A and III B. Next, we tackle the second regime in Sec. III C.

\section{A. Zel'dovich power spectrum}

If all particle pairs can be described by the same perturbative framework, the power spectrum [Eq.(6)] can be written as

$$
\begin{aligned}
P(k) & =\int \frac{\mathrm{d} \Delta \mathbf{q}}{(2 \pi)^{3}}\left\langle e^{\mathrm{i} \mathbf{k} \cdot \Delta \mathbf{x}}\right\rangle \\
& =\int \frac{\mathrm{d} \Delta \mathbf{q}}{(2 \pi)^{3}} \exp \left[\sum_{n=1}^{\infty} \frac{\left\langle(\mathrm{ik} \cdot \Delta \mathbf{x})^{n}\right\rangle_{c}}{n !}\right] .
\end{aligned}
$$

In the first line we used the fact that the integral of the last term in Eq. (6) gives a Dirac factor $\delta_{D}(\mathbf{k})$ that can be discarded for $k>0$, and in the second line we used the usual expansion over cumulants.

In the well-known Zel'dovich approximation [23], we use the linear prediction for the Eulerian separation $\Delta \mathbf{x}$. Then, for Gaussian initial conditions, only the first- and second-order cumulants are nonzero. Introducing the displacement field $\boldsymbol{\Psi}, \mathbf{x}(\mathbf{q}, t)=\mathbf{q}+\mathbf{\Psi}(\mathbf{q}, t)$, at linear order we have the longitudinal and transverse variances (with respect to the direction defined by the initial Lagrangian separation $\Delta \mathbf{q})$ :

$$
\begin{aligned}
\sigma_{\|}^{2}(\Delta q) & =2 \int \mathrm{d} \mathbf{k}\left[1-\cos \left(k_{1} \Delta q\right)\right] \frac{k_{1}^{2}}{k^{4}} P_{L}(k), \\
\sigma_{\perp}^{2}(\Delta q) & =2 \int \mathrm{d} \mathbf{k}\left[1-\cos \left(k_{1} \Delta q\right)\right] \frac{k_{2}^{2}}{k^{4}} P_{L}(k) .
\end{aligned}
$$

Here we took $\Delta \mathbf{q}=(\Delta q) \mathbf{e}_{1}$ along the first axes, $\mathbf{e}_{1}$, and we labeled the two transverse axis as $\mathbf{e}_{2}$ and $\mathbf{e}_{3}$. Thus, $\sigma_{\|}^{2}=\left\langle\left(\Delta \Psi_{L \|}\right)^{2}\right\rangle=\left\langle\left(\Delta \Psi_{L 1}\right)^{2}\right\rangle$ and $\sigma_{\perp}^{2}=\left\langle\left(\Delta \Psi_{L 2}\right)^{2}\right\rangle=$ 


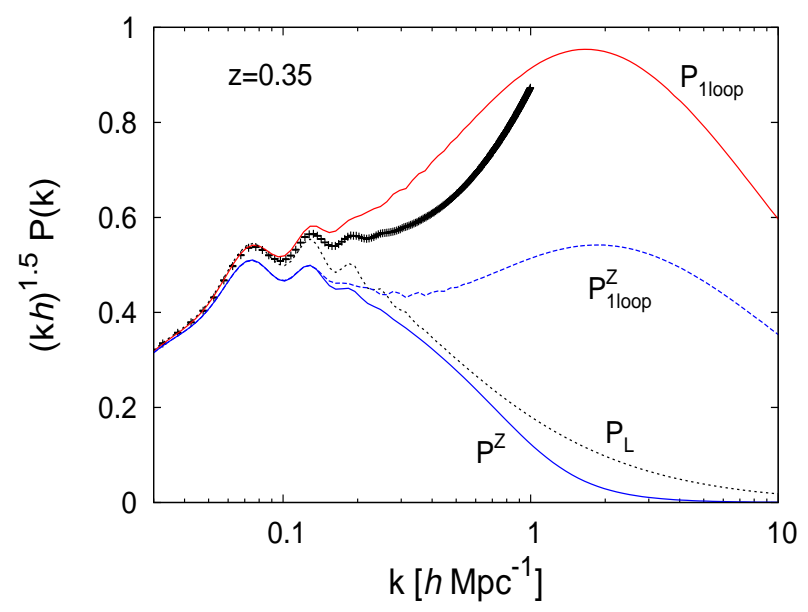

FIG. 1: Matter density power spectrum at $z=0.35$ (multiplied by a factor $(k h)^{1.5}$ to decrease the vertical range). We show the results from N-body simulations (data points), the linear power spectrum $P_{L}$, the standard one-loop prediction $P_{1 \text { loop }}$, the one-loop prediction $P_{1 \text { loop }}^{Z}$ (18) within the Zel'dovich approximation, and the full nonlinear Zel'dovich power spectrum $P^{\mathrm{Z}}$ (16).

$\left\langle\left(\Delta \Psi_{L 3}\right)^{2}\right\rangle$. Introducing the integrals

$$
I_{\ell}(q)=\frac{4 \pi}{3} \int_{0}^{\infty} \mathrm{d} k P_{L}(k) j_{\ell}(q k)
$$

and the variance of the linear one-point displacement along one dimension,

$$
\sigma_{v}^{2}=\frac{1}{3}\left\langle\left|\mathbf{\Psi}_{L}(\mathbf{q})\right|^{2}\right\rangle=I_{0}(0),
$$

we have:

$$
\begin{aligned}
\sigma_{\|}^{2}(\Delta q) & =2 \sigma_{v}^{2}-2 I_{0}(\Delta q)+4 I_{2}(\Delta q), \\
\sigma_{\perp}^{2}(\Delta q) & =2 \sigma_{v}^{2}-2 I_{0}(\Delta q)-2 I_{2}(\Delta q) .
\end{aligned}
$$

Then, in the Zel'dovich approximation, where $\mathbf{x}=\mathbf{q}+$ $\boldsymbol{\Psi}_{L}$, Eq.(9) reads as

$$
P^{\mathrm{Z}}(k)=\int \frac{\mathrm{d} \Delta \mathbf{q}}{(2 \pi)^{3}} e^{\mathrm{i} k \mu \Delta q-\frac{1}{2} k^{2} \mu^{2} \sigma_{\|}^{2}-\frac{1}{2} k^{2}\left(1-\mu^{2}\right) \sigma_{\perp}^{2}},
$$

where $\mu=(\mathbf{k} \cdot \Delta \mathbf{q}) /(k \Delta q)$. (By symmetry, we always have $\langle\Delta \Psi\rangle=0$.) At large distances, the linear displacements $\boldsymbol{\Psi}\left(\mathbf{q}_{1}\right)$ and $\boldsymbol{\Psi}\left(\mathbf{q}_{2}\right)$ of the two particles become independent and $\sigma_{\|}^{2}$ and $\sigma_{\perp}^{2}$ converge to $2 \sigma_{v}^{2}$, in agreement with Eqs.(14)-(15). Then, Eq.(16) is formally divergent at large $q$, as it contains a Dirac factor $\delta_{D}(\mathbf{k})$, and for numerical computations it is convenient to compute in a separate fashion the low-order terms.

By expanding the exponential (9) over powers of $P_{L}$, one recovers the standard Eulerian perturbation theory.
Within the Zel'dovich approximation, we obtain, for instance, up to second order [24],

$$
P^{Z}(k)=P_{L}(k)+P_{1 \text { loop }}^{Z}(k)+\ldots
$$

with

$$
\begin{aligned}
P_{\text {1loop }}^{\mathrm{Z}}(k)= & -k^{2} \sigma_{v}^{2} P_{L}(k)+\int \mathrm{d} \mathbf{k}_{1} \mathrm{~d} \mathbf{k}_{2} \delta_{D}\left(\mathbf{k}_{1}+\mathbf{k}_{2}-\mathbf{k}\right) \\
& \times \frac{\left(\mathbf{k} \cdot \mathbf{k}_{1}\right)^{2}\left(\mathbf{k} \cdot \mathbf{k}_{2}\right)^{2}}{2 k_{1}^{4} k_{2}^{4}} P_{L}\left(k_{1}\right) P_{L}\left(k_{2}\right) .
\end{aligned}
$$

This is different from the exact one-loop contribution $P_{1 \text { loop }}$ generated by the actual gravitational dynamics, because at this order we should include the third-order cumulants and loop corrections to the second-order cumulants (the cumulant of order $n$ scales as $\left\langle(\Delta \Psi)^{n}\right\rangle_{c} \propto$ $P_{L}^{n-1}$ at leading order).

We compare in Fig. 1 the power spectra obtained from the true gravitational dynamics (at linear and oneloop order, as well as the full nonlinear power spectrum from N-body simulations) with those associated with the Zel'dovich approximation (at one-loop order and for the full nonlinear power spectrum). As is well known, the standard Eulerian one-loop prediction $P_{1 \text { loop }}$ improves the agreement with simulations as compared with linear theory on large scales, but quickly gives too much power and is badly behaved at high $k$. In contrast, the nonlinear Zel'dovich power spectrum $P^{Z}$ decays faster than the linear power at high $k$ 21, 22]. For instance, for a power-law linear power spectrum $P_{L}(k) \propto k^{n}$ with $-3<n<-1$, we have $P^{\mathrm{Z}}(k) \sim k^{-3+3(n+3) /(n+1)}$ at high $k$ [2]. This is due to the fact that within the approximation of linear trajectories, particles keep moving along straight lines after shell crossing, so that pancakes and filaments quickly fatten and dissolve after their formation. This also leads to a one-loop Zel'dovich prediction (18) that is smaller than the linear power spectrum on weakly nonlinear scales, where it is meaningful and follows the full nonlinear power $P^{\mathrm{Z}}$. Therefore, at one-loop order, the Zel'dovich approximation is actually worse than the simple linear approximation.

Figure 1 teaches us two things if we wish to describe the power spectrum in a Lagrangian framework:

(1) We must explicitly modify the Zel'dovich approximation at one-loop order to (partly) cure the underestimation of the power spectrum on weakly nonlinear scales.

(2) We must modify the behavior after shell crossing to cure the high- $k$ damping.

\section{B. Perturbative Lagrangian ansatz}

\section{Factorized ansatz}

Before tackling the high- $k$ regime in Sec. IIIC (point 2 above), we address the weakly nonlinear regime in this section (point 1), where we disregard shell crossing. Thus, we look for a Lagrangian ansatz that improves over 
(16) on weakly nonlinear scales and shows a perturbative expansion over integer powers of $P_{L}$ as in the true gravitational dynamics.

By symmetry, the means $\left\langle\left(\Delta \Psi_{\|}\right)^{2} \Delta \boldsymbol{\Psi}_{\perp}\right\rangle_{c}$ and $\left\langle\left(\Delta \boldsymbol{\Psi}_{\perp}\right)^{3}\right\rangle_{c}$ are zero, and at order $P_{L}^{2}$ we are left with the two third-order cumulants, $\left\langle\left(\Delta \Psi_{\|}\right)^{3}\right\rangle_{c}$ and $\left\langle\Delta \Psi_{\|}\left(\Delta \Psi_{\perp}\right)^{2}\right\rangle_{c}$. In particular, the latter average shows that beyond the Gaussian order (16), the longitudinal and transverse displacements are generically correlated. Nevertheless, because our goal is to build a simple ansatz for Eq. (9), we consider a simplified model where the longitudinal and transverse displacements are uncorrelated. Then, since $\left\langle\Delta \Psi_{\perp}^{4}\right\rangle_{c}$ is of order $P_{L}^{3}$, we keep the linear Gaussian for the transverse part and look for an ansatz of the form

$$
P^{\|}(k)=\int \frac{\mathrm{d} \Delta \mathbf{q}}{(2 \pi)^{3}}\left\langle e^{\mathrm{i} k \mu \Delta x_{\|}}\right\rangle_{\|} e^{-\frac{1}{2} k^{2}\left(1-\mu^{2}\right) \sigma_{\perp}^{2}},
$$

where $\Delta x_{\|}=\Delta q+\Delta \Psi_{\|}$is the longitudinal Eulerian separation and we need to specify a model for the mean $\langle. .\rangle_{\|}$. Thus, the difference from the Zel'dovich approximation (16) is that we go beyond the Gaussian for the longitudinal part. Apart from simplicity and ordering in perturbation theory, the reason why we focus on the longitudinal part is that it should be more sensitive than the transverse part to the progress of nonlinear clustering, even at a qualitative level. Indeed, by symmetry, the probability distribution of the relative displacement along any transverse direction, $\mathcal{P}\left(\Delta \Psi_{\perp}\right)$, remains even and peaks at $\Delta \Psi_{\perp}=0$, so that on a qualitative level its shape remains similar to a Gaussian. In contrast, the probability distribution of the longitudinal Eulerian separation, $\mathcal{P}\left(\Delta x_{\|}\right)$, which in the linear regime is a Gaussian centered on $\Delta x_{\|}=\Delta q$, develops an asymmetry (skewness) that is characteristic of the gravitational dynamics. Moreover, following [19], negative values of $\Delta x_{\|}$are expected to be closely associated with shell crossing, so that keeping track of $\Delta x_{\|}$will be useful to handle shellcrossing effects, as explained in Sec. IIIC below.

\section{One-loop order}

It is convenient to define the dimensionless Eulerian longitudinal separation $\kappa_{\|}$by

$$
\kappa_{\|}=\frac{\Delta x_{\|}}{\Delta q}=1+\frac{\Delta \Psi_{\|}}{\Delta q}
$$

and its linear variance and its skewness by

$$
\sigma_{\kappa_{\|}}^{2}=\frac{\sigma_{\|}^{2}}{(\Delta q)^{2}}, \quad S_{3}^{\kappa_{\|}}=\frac{\left\langle\kappa_{\|}^{3}\right\rangle_{c}}{\left\langle\kappa_{\|}^{2}\right\rangle_{c}^{2}}=(\Delta q) \frac{\left\langle\left(\Delta \Psi_{\|}\right)^{3}\right\rangle_{c}}{\left\langle\left(\Delta \Psi_{\|}\right)^{2}\right\rangle_{c}^{2}} .
$$

We show the linear variances $\sigma_{\|}$and $\sigma_{\kappa_{\|}}$[as well as the transverse counterparts $\sigma_{\perp}$ and $\left.\sigma_{\kappa_{\perp}}=\sigma_{\perp} /(\Delta q)\right]$ in Fig. 2. We can check that above $3 h^{-1} \mathrm{Mpc}$, at $z=0.35$,

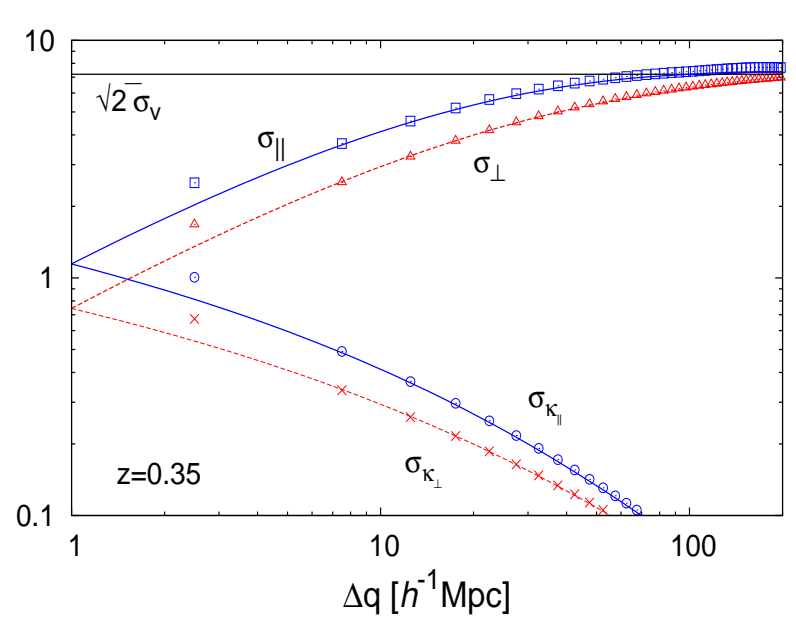

FIG. 2: The linear variances $\sigma_{\|}$and $\sigma_{\perp}$ (in units of $h^{-1} \mathrm{Mpc}$ ), and $\sigma_{\kappa}$, at $z=0.35$. The lines are the linear theory predictions (10)-(11), and the points are the results from N-body simulations.

linear theory gives a good estimate of these variances. On smaller scales, nonlinearities and nonperturbative effects (virialized motions in the shell-crossing regime), which we do not consider in this section, increase the amplitude of these rms displacements. As noticed above, at large distances $\sigma_{\|}$and $\sigma_{\perp}$ converge to $\sqrt{2} \sigma_{v}$, because the motions of the two particles become independent. This implies that $\sigma_{\kappa}$ decreases as $1 /(\Delta q)$ on large scales. On small separations, for CDM power spectra that decrease faster than $k^{-3}$ at high $k, \sigma_{\|}$and $\sigma_{\perp}$ decrease as $\Delta q$, and $\sigma_{\kappa}$ converges to a constant. However, we can see that these asymptotic regimes are only reached on very large or very small scales. The value of $\sigma_{\kappa}$ is a better measure of nonlinearity than the rms displacements $\sigma_{\|}$ and $\sigma_{\perp}$, and $\sigma_{\kappa} \sim 1$ marks the transition to the nonlinear regime. From the definition (20), in a spherical dynamics $\kappa_{\|} \sim(1+\delta)^{-1 / 3}$, and deviations of order unity of $\kappa_{\|}$from its mean are associated with density contrasts of order unity. In particular, $\kappa_{\|}-1=-1$ corresponds to shell crossing and infinite density. In CDM cosmologies, smaller scales turn nonlinear first, and we can check in Fig. 2 that $\sigma_{\kappa}$ increases on smaller scales.

To further simplify our ansatz, we keep the linear theory prediction $\sigma_{\kappa_{\|}}^{2}$ for the variance $\left\langle\kappa_{\|}^{2}\right\rangle_{c}$. Then, the only difference at order $P_{L}^{2}$ of the power spectrum (19) from the Zel'dovich approximation (16) arises from the skewness $S_{3}^{\kappa_{\|}}$:

$$
P_{1 \text { loop }}^{\|}(k)=P_{1 \text { loop }}^{Z}(k)+P_{S_{3}}(k),
$$

with

$$
P_{S_{3}}(k)=\int \frac{\mathrm{d} \Delta \mathbf{q}}{(2 \pi)^{3}} \sin (k \mu \Delta q)(k \mu \Delta q)^{3} \frac{S_{3}^{\kappa} \| \sigma_{\kappa_{\|}}^{4}}{6} .
$$


The integration over angles gives

$$
\begin{aligned}
P_{S_{3}}(k) & =\int_{0}^{\infty} \frac{\mathrm{d} \Delta q}{2 \pi^{2}}(\Delta q)^{2} \frac{S_{3}^{\kappa_{\|}} \sigma_{\kappa_{\|}}^{4}}{6}\left\{\left[6-(k \Delta q)^{2}\right]\right. \\
& \left.\times \cos (k \Delta q)+\left[(k \Delta q)^{2}-2\right] 3 \frac{\sin (k \Delta q)}{k \Delta q}\right\} .
\end{aligned}
$$

Then, to complete our model at one-loop order, we must set the skewness $S_{3}^{\kappa}$. A first choice would be to use the prediction from lowest-order perturbation theory. Then, going up to second order in Lagrangian perturbation theory ("2LPT", see 25 27]), we obtain

$$
\begin{aligned}
& S_{3}^{\kappa_{\|}, \text {P.T. }}(\Delta q)=\frac{18}{7 \sigma_{\kappa_{\|}}^{4}} \int \mathrm{d} \mathbf{k}_{1} \mathrm{~d} \mathbf{k}_{2} P_{L}\left(k_{1}\right) P_{L}\left(k_{2}\right) \\
& \quad \times \frac{k_{1 \|} k_{2 \|}\left(k_{1 \|}+k_{2 \|}\right)}{k_{1}^{2} k_{2}^{2}\left|\mathbf{k}_{1}+\mathbf{k}_{2}\right|^{2}(\Delta q)^{3}}\left[1-\frac{\left(\mathbf{k}_{1} \cdot \mathbf{k}_{2}\right)^{2}}{k_{1}^{2} k_{2}^{2}}\right] \\
& \quad \times\left\{\sin \left[\left(k_{1 \|}+k_{2 \|}\right) \Delta q\right]-\sin \left(k_{1 \|} \Delta q\right)-\sin \left(k_{2 \|} \Delta q\right)\right\} .
\end{aligned}
$$

However, this choice implies that the power spectrum $P^{\|}(k)$ only agrees with perturbation theory at the linear level. Indeed, it misses the contribution from the crosscorrelation $\left\langle\Delta \Psi_{\|}\left(\Delta \boldsymbol{\Psi}_{\perp}\right)^{2}\right\rangle_{c}$ and one-loop contributions to the variances $\left\langle\left(\Delta \Psi_{\|}\right)^{2}\right\rangle_{c}$ and $\left\langle\left(\Delta \boldsymbol{\Psi}_{\perp}\right)^{2}\right\rangle_{c}$. In principle, this could be included by building an ansatz for the bivariate distribution $\mathcal{P}\left(\Delta \Psi_{\|}, \Delta \Psi_{\perp}\right)$ that does not factorize and is parameterized by the exact second and third cumulants (up to one-loop order). In this spirit, one may include perturbative results up to any finite order, at the price of increasingly complex models for $\mathcal{P}\left(\Delta \Psi_{\|}, \Delta \boldsymbol{\Psi}_{\perp}\right)$.

Our approach in this paper follows a different strategy. We consider Eq. (19) as a qualitative ansatz that allows us to build a simple model that is physically consistent and provides a good behavior in all regimes, as we explain below. Then, we treat the parameters of this ansatz as free parameters that we set so as to match the exact perturbative expansion up to the required order. In this paper we only go up to one-loop order (i.e., $P_{L}^{2}$ ), which means that we choose $S_{3}^{\kappa \|}$ so that $P_{1 \text { loop }}^{\|}(k)=P_{1 \text { loop }}(k)$, that is,

$$
P_{S_{3}}(k)=P_{1 \text { loop }}(k)-P_{1 \text { loop }}^{\mathrm{Z}}(k),
$$

where $P_{1 \text { loop }}$ is the exact one-loop power spectrum given by standard perturbation theory. Inverting Eq.(24) this gives the effective skewness

$$
\begin{aligned}
S_{3}^{\kappa_{\|}, \text {eff. }}(\Delta q)= & -\frac{24 \pi}{\sigma_{\kappa_{\|}}^{4}} \int_{0}^{\infty} \mathrm{d} k \frac{P_{\text {1loop }}(k)-P_{\text {lloop }}^{Z}(k)}{(\Delta q)^{4} k^{2}} \\
& \times\left[2+\cos (k \Delta q)-3 \frac{\sin (k \Delta q)}{k \Delta q}\right] .
\end{aligned}
$$

The explicit expression (27) allows us to recover the exact one-loop power spectrum for any cosmology. To be meaningful, such an approach requires that we start from an ansatz that is not too far from the exact dynamics.

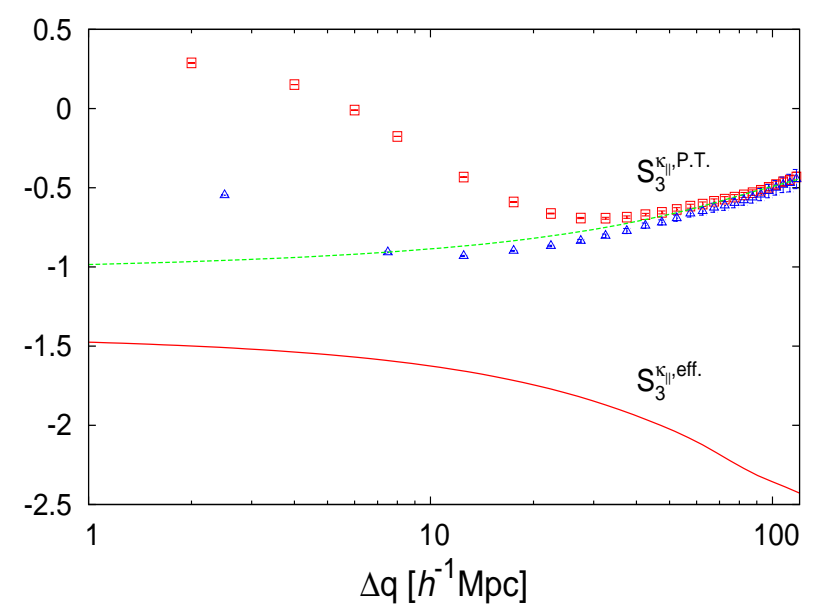

FIG. 3: The skewness $S_{3}^{\kappa} \|$ given by lowest-order perturbation theory [Eq. (25), upper dashed line] and by the one-loop power-spectrum matching [Eq.(27), lower solid line]. The points are the results from N-body simulations at $z=0.35$ (squares) and $z=2$ (triangles)

This is indeed the case on a qualitative level, as Eqs.(25) and (27) show similar functional forms: in both cases $\left(S_{3}^{\kappa_{\|}} \sigma_{\kappa_{\|}}^{4}\right)$ is a quadratic integral over $P_{L}$, and $S_{3}^{\kappa_{\|}}$is independent of redshift and of the amplitude of the linear power spectrum. On a quantitative level, the comparison in Fig. 3 shows that the effective skewness is greater (in amplitude) than the perturbative skewness by a factor of about 2 and that it has the same sign.

Of course, if one is interested in the statistics of $\kappa_{\|}$for its own sake, one should use the perturbative prediction (25) rather than the effective value (27). In particular, Fig. [3] shows that the skewness is well described by lowestorder perturbation theory above $15 h^{-1} \mathrm{Mpc}$, at $z=0.35$. (On small scales, the skewness measured in the simulations increases and becomes positive because of nonlinearities and nonperturbative effects. As expected, this upturn shifts to larger scales at lower redshift.)

The effective value (27) only makes sense as an ingredient for a model for the power spectrum. Thus, we also show in Fig. 4 the one-loop power spectrum (22) that would be obtained using the perturbative skewness (25). This yields a power spectrum $P_{1 \text { loop }}^{\|, \text {P.T. }}$ that is halfway between the Zel'dovich and gravitational one-loop power spectra and much closer to the N-body data. Therefore, it already provides a more realistic starting point than the Zel'dovich approximation. Then, modifying the skewness as in Eq.(27) to recover the exact one-loop power spectrum is not a very large modification, and we can hope that the ansatz determined by Eqs. (19) and (27) remains sufficiently close to the exact dynamics to be useful. 


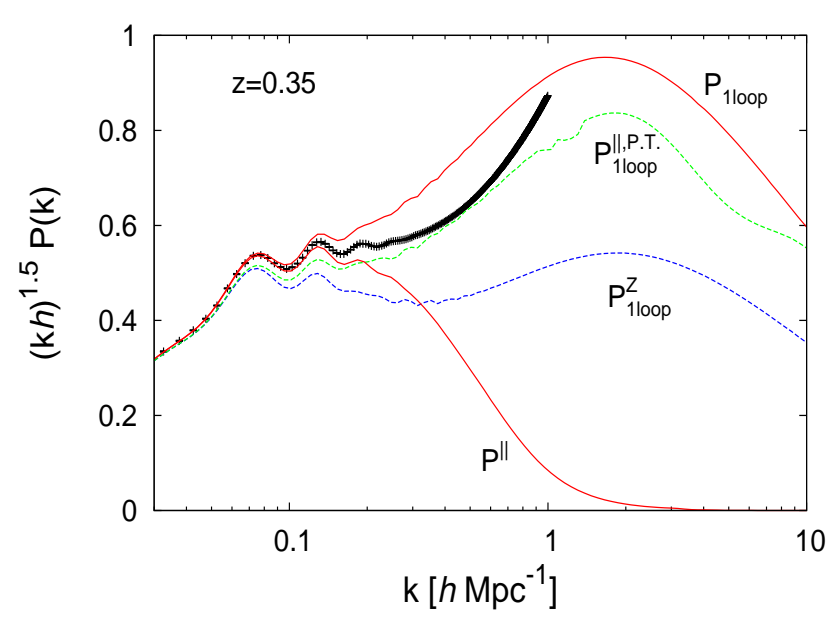

FIG. 4: Matter density power spectrum at $z=0.35$, as in Fig. 1. We show the results from N-body simulations (data points), the standard one-loop prediction $P_{1 \text { loop }}$, the one-loop prediction $P_{1 \text { loop }}^{\mathrm{Z}}$ (18) within the Zel'dovich approximation, the one-loop prediction $P_{\text {1loop }}^{\|, P . T .}(22)$ using the perturbative skewness (25), and the full nonlinear prediction $P^{\|}$of Eq. (35) using the effective skewness (27).

\section{Resummed ansatz}

The one-loop power spectrum (22) was obtained by expanding the exponential (9) up to order $P_{L}^{2}$, which only involves the second and third cumulants. However, one of the main goals of this paper is precisely not to expand the exponential (9). Indeed, it is well known that no probability distribution exists such that all its cumulants vanish beyond some order $n>3$ (i.e., the only distribution with a finite set of nonzero cumulants is the Gaussian). In particular, approximations such as the Gram-Charlier or Edgeworth expansions, where we expand up to some finite order over cumulants, lead to functions that are not guaranteed to be positive and may not be valid probability distributions. Of course, this is not necessarily a problem, if one restricts oneself to the range of validity of such approximations. However, the spirit of this paper is to avoid as far as possible such inconsistencies and to develop an approach that remains well behaved in all regimes.

Indeed, we can hope that by satisfying such constraints, the convergence of our scheme will be improved and the matching to the highly nonlinear regime will be smoother. In particular, this should avoid the bad behavior encountered in the standard Eulerian perturbative expansion, where higher orders improve the accuracy on very large scales but yield increasingly divergent quantities on small scales. Thus, the rise of $P_{1 \text { loop }}^{\|}$at high $k$ in Fig. 4 around $k>0.5 h / \mathrm{Mpc}$, is an artifact due to the truncation at one-loop order. As for the Eulerian perturbative expansion, higher orders will also give large contributions on these scales, and the much smaller full nonlinear power spectrum will result from a compensation between these much larger terms. [Indeed, as explained in point 2 in Sec. IIA we know that the logarithmic nonlinear power spectrum $k^{3} P(k)$ should not grow much beyond the nonlinear scale as long as the shellcrossing regime has not been drastically modified from the Zel'dovich dynamics by trapping particles in smallscale structures.] This means that the perturbative expansion has a very slow convergence at best (provided it is convergent, which is not always the case [2]), and that including an increasing number of higher-order terms is not sufficient to build a useful model for our purposes.

To resum all cumulants, it is convenient to define the cumulant-generating function $\varphi_{\|}(y)$ of $\kappa_{\|}$as

$$
e^{-\varphi_{\|}(y) / \sigma_{\kappa}^{2} \|}=\left\langle e^{-y \kappa_{\|} / \sigma_{\kappa}^{2} \|}\right\rangle_{\|}
$$

which can be expanded at $y=0$ as

$$
\varphi_{\|}(y)=-\sum_{n=1}^{\infty} \frac{S_{n}^{\kappa_{\|}}}{n !}(-y)^{n}, \quad S_{n}^{\kappa_{\|}}=\frac{\left\langle\kappa_{\|}^{n}\right\rangle_{c}}{\sigma_{\kappa_{\|}}^{2(n-1)}} .
$$

(This function is always well defined by the average (28), at least along the whole imaginary axis, even when the series (29) is divergent or cumulants beyond a finite order do not exist.) The choice of the generating function $\varphi_{\|}$ will define our ansatz (19). From the Taylor expansion (29), we have the behavior at the origin

$$
y \rightarrow 0: \quad \varphi_{\|}(y)=y-\frac{y^{2}}{2}+S_{3}^{\kappa_{\|}} \frac{y^{3}}{6}+\ldots
$$

Defining the probability distribution $\mathcal{P}_{\|}\left(\kappa_{\|}\right)$through its cumulant-generating function $\varphi_{\|}(y)$ shows several advantages. First, imposing the expansion (30) up to order $y^{3}$ at the origin automatically ensures that the probability distribution is normalized to unity, its mean is $\left\langle\kappa_{\|}\right\rangle=1$, its variance $\sigma_{\kappa_{\|}}^{2}$, and its skewness $S_{3}^{\kappa_{\|}}$. Second, choosing coefficients $S_{n}^{\kappa_{\|}}$(i.e., a function $\varphi_{\|}$) that do not depend on time or the amplitude of the linear power spectrum automatically ensures the scaling over $P_{L}$ given by perturbation theory (at leading order). Indeed, as we have already noticed above, in perturbation theory $\left\langle\kappa_{\|}^{n}\right\rangle_{c}$ scales as $P_{L}^{n-1}$. This ensures that the power spectrum (19) can be expanded over integer powers of $P_{L}$ as in the exact perturbation theory (but of course, the exact value of each term will not be reproduced by our simple model). Third, from Eq.(28), we can see that the average $\langle. .\rangle_{\|}$that enters Eq.(19) has an explicit expression in terms of $\varphi_{\|}$. This avoids performing an additional integration over the probability distribution $\mathcal{P}_{\|}\left(\kappa_{\|}\right)$, which is convenient for numerical purposes. In this paper, we choose the following ansatz for $\varphi_{\|}(y)$,

$$
\varphi_{\|}(y)=\frac{1-\alpha}{\alpha}\left(1+\frac{y}{1-\alpha}\right)^{\alpha}-\frac{1-\alpha}{\alpha},
$$




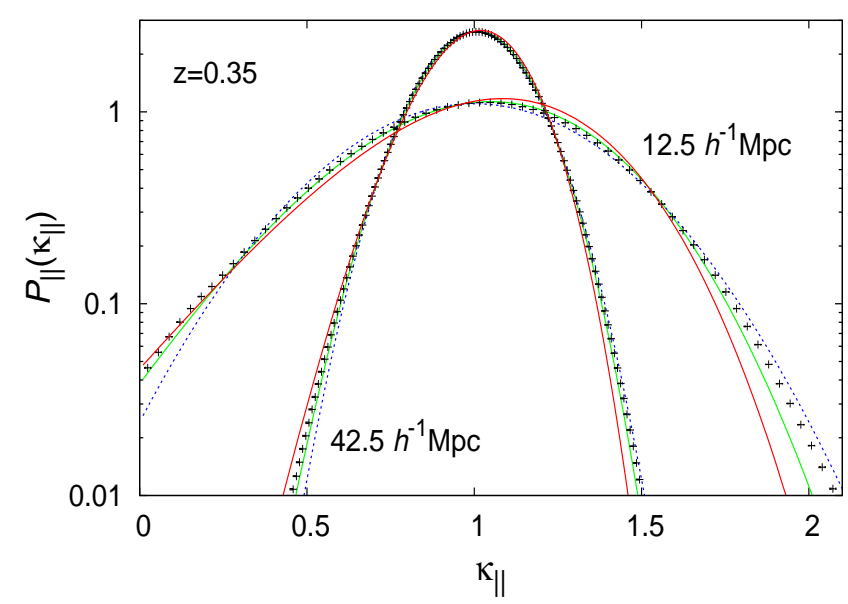

FIG. 5: The probability distribution of the longitudinal relative displacement, $\kappa_{\|}=\Delta x_{\|} / \Delta q$, at $z=0.35$, for the Lagrangian separations $\Delta q=12.5 h^{-1} \mathrm{Mpc}$ and $\Delta q=$ $42.5 h^{-1} \mathrm{Mpc}$. We show the linear-theory Gaussian (dotted line) and our model (34) (solid lines), using for the skewness either Eq. (25) (intermediate curve) or (27) (largest departure from the Gaussian). The points are the results from N-body simulations.

where the scale-dependent parameter $\alpha(\Delta q)$ is set from Eq.(30),

$$
S_{3}^{\kappa \|}=\frac{\alpha-2}{\alpha-1} .
$$

This gives the dependence of $\varphi_{\|}$on the scale $\Delta q$ through

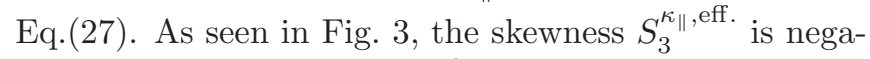
tive, hence the parameter $\alpha$ falls in the range

$$
1<\alpha \leq 2 \text {. }
$$

The case of a zero skewness corresponds to $\alpha=2$, where the generating function (31) becomes the quadratic polynomial $\varphi_{\|}(y)=y-y^{2} / 2$, and we recover the Gaussian case (i.e., the Zel'dovich approximation (16)).

The power law (31) is the simplest function that obeys the constraint (30) while being a valid cumulantgenerating function. [This requires, for instance, that $-\varphi_{\|}(y)$ be convex.] Thus, it provides a simple continuation of the series of cumulants: instead of truncating at third order (i.e., at the skewness), we automatically generate all higher-order cumulants in a manner that provides a meaningful probability distribution $\mathcal{P}_{\|}\left(\kappa_{\|}\right)$in all regimes (i.e., $\mathcal{P}_{\|}\left(\kappa_{\|}\right)$is always positive, normalized to unity, and with a mean $\left.\left\langle\kappa_{\|}\right\rangle=1\right)$. From Eq.(28), this "perturbative" probability distribution $\mathcal{P}_{\|}\left(\kappa_{\|}\right)$is given by the inverse Laplace transform

$$
\mathcal{P}_{\|}\left(\kappa_{\|}\right)=\int_{-\mathrm{i} \infty}^{+\mathrm{i} \infty} \frac{\mathrm{d} y}{2 \pi \mathrm{i} \sigma_{\kappa_{\|}}^{2}} e^{\left[\kappa y-\varphi_{\|}(y)\right] / \sigma_{\kappa_{\|}}^{2}} .
$$

Because the skewness $S_{3}^{\kappa} \|$ is negative, the right tail is sharper than the left tail, as can be checked in Fig. 5. In agreement with Fig. 3. Fig. 5 shows that using the perturbative prediction (25), with the ansatz (31), provides a good match to the full probability distribution measured on large scales in N-body simulations. As expected, using the larger value (27) for the skewness amplifies the deviation from the Gaussian and yields a distribution that is somewhat too skewed towards the left. However, the point of Fig. 5 is only to check that our model, defined by Eqs. (27) and (31), provides a physically consistent and realistic ansatz. This should be understood as a simpler effective system that is not expected to simultaneously reproduce with the same accuracy all statistical properties of the dynamics. Thus, depending on whether one is interested in $\mathcal{P}_{\|}\left(\kappa_{\|}\right)$or in $P(k)$, one should use either Eq.(25) or (27).

In both cases, the skewness is negative, which can be understood from the effects of gravity, as compared with the Zel'dovich dynamics (associated with the linear theory Gaussian). Indeed (considering for instance a spherical configuration), particles that move outward $\left(\kappa_{\|}>1\right)$ will be slowed down by gravity until they turn around and eventually merge into a single halo. This leads to an Eulerian separation that is smaller than the one predicted by linear theory, whence a sharper cutoff of the probability distribution $\mathcal{P}_{\|}\left(\kappa_{\|}\right)$for large positive $\kappa_{\|}$. In contrast, particles that move inward will be accelerated by gravity (because of the $1 / r^{2}$ behavior of the three-dimensional gravity). This leads to a smaller value of the Eulerian separation than the linear prediction, which now gives a more extended tail towards low values of $\kappa_{\|}$, below the static value $\kappa_{\| \text {,static }}=1$. Of course, this argument does not extend to $\kappa_{\|}<0$, where shell crossing and changes of direction within virialized halos are expected.

The spirit of our approach is different from resummation schemes, where one performs partial resummations of higher-order terms by computing a subset of higherorder Feynman diagrams [5, 6, 10, 28] or expanding over some auxiliary parameter [2, 7] (such as the dimension of space $d$ or the number of field components $N$ ). Here we do not explicitly compute approximations to $S_{n}^{\kappa_{\|}}$for $n \geq 4$ from a subset of diagrams, as these coefficients are automatically generated by the functional form (31) from the low-order ones. (Here we only take $S_{3}^{\kappa_{\|}}$as input, but as in resummed perturbative approaches, we could exactly include all terms up to order $n$ and generate approximations for higher orders by using an ansatz for $\varphi_{\|}$ that is parameterized by the set $\left\{S_{3}^{\kappa_{\|}}, . ., S_{n}^{\kappa_{\|}}\right\}$.) The advantage of our approach is that it ensures a well-behaved "resummed" (or rather "regularized") probability distribution $\mathcal{P}_{\|}\left(\kappa_{\|}\right)$, while explicit perturbative approaches do not always guarantee that their resummation is physically consistent.

As explained in the Introduction, we can hope that by ensuring physical consistency, our method will be better behaved and show a faster convergence. In this approach we only ensure self-consistency up to a partial degree, in 
terms of the probability distribution $\mathcal{P}_{\|}\left(\Delta \Psi_{\|}\right)$, but we do not show that physical density fields can exactly realize such a probability distribution, which is a more difficult problem. Nevertheless, this is already a significant improvement over previous Lagrangian-space methods that expand the exponential (91) and truncate at some finite order [16, 17, 29].

Then, Eqs.(31), (32), and (27) fully define the power spectrum (19). Using Eq.(28), this yields

$$
P^{\|}(k)=\int \frac{\mathrm{d} \Delta \mathbf{q}}{(2 \pi)^{3}} e^{-\varphi_{\|}(-y) / \sigma_{\kappa}^{2}} \| e^{-\frac{1}{2} k^{2}\left(1-\mu^{2}\right) \sigma_{\perp}^{2}},
$$

where we denote

$$
y=\mathrm{i} k \mu \Delta q \sigma_{\kappa_{\|}}^{2} .
$$

We recover the Zel'dovich approximation (16) for $\varphi_{\|}(y)=y-y^{2} / 2$, which corresponds to $\alpha=2$ and $S_{3}^{\kappa \|}=0$ in Eqs.(31) and (32). Thus, we can see that our ansatz (35) is a continuous generalization of the Zel'dovich power spectrum, parameterized by the skewness $S_{3}^{\kappa \|}$. In agreement with a well-known mathematical result, it is only in the Gaussian case (i.e., the Zel'dovich approximation) that the cumulant-generating function is a finite-order polynomial.

As seen in Fig. 4, the power spectrum (35) follows the one-loop power (22) on large scales, which is identical to the exact one-loop power spectrum because we use the skewness (27), and it improves over the Zel'dovich approximation (compare with the Zel'dovich one-loop power $P_{1 \text { loop }}^{Z}$ or with Fig. (1). At high $k, P^{\|}(k)$ converges back to a behavior similar to the Zel'dovich power spectrum, due to the fact that particles still escape to infinity after shell crossing. Although we will modify this behavior in the next section, to include the building of pancakes in a simplified form, this shows the main property that we looked for in this section: the power spectrum remains well behaved at high $k$, with a universal behavior that does not change with the order of the perturbation theory up to which one requires consistency.

The perturbative expansion of the power spectrum (35) over powers of the linear power spectrum $P_{L}$ is likely to be divergent for $k>k^{\max }$, with

$$
k^{\max }=\min _{\Delta q}\left[\frac{\alpha-1}{\Delta q \sigma_{\kappa_{\|}}^{2}}\right],
$$

because of the finite radius of convergence of the generating function $\varphi_{\|}(y)$ around $y=0$. This gives $k^{\max } \sim$ $0.2 h \mathrm{Mpc}^{-1}$ at $z=0.35$ (and $k^{\max }$ decreases with time as $\left.1 / \sigma_{\kappa_{\|}}^{2}\right)$. This does not necessarily apply to the actual power spectrum built by the gravitational dynamics, and these values would be modified by using different generating functions than (31). However, it explicitly shows a possible limitation of some perturbative approaches and the importance of choosing appropriate expansions or resummations. This is independent of the limitation due to shell crossing and only due to the fast growth of highorder cumulants shown by our ansatz.

The power spectrum (35) is closely related to Lagrangian perturbation theory. Indeed, it can be expanded over integer powers of $P_{L}$, and it is based on a partial implicit resummation of higher-order cumulants $\left\langle\left(\Delta \Psi_{\|}\right)^{n}\right\rangle_{c}$, which scale at leading order over $P_{L}$ as in perturbation theory. For simplicity, we have only included the linear theory variances $\left\langle\left(\Delta \Psi_{\|}\right)^{2}\right\rangle$ and $\left\langle\left(\Delta \boldsymbol{\Psi}_{\perp}\right)^{2}\right\rangle$ and the third-order cumulant $\left\langle\left(\Delta \Psi_{\|}\right)^{3}\right\rangle_{c}$, which is set by the matching with the exact one-loop contribution to the power spectrum. However, this approach could be made exact up to an arbitrary order of perturbation theory by including loop contributions to these quantities and higher-order cumulants, such as $\left\langle\left(\Delta \Psi_{\|}\right)^{4}\right\rangle_{c}$, and cross-correlations, such as $\left\langle\Delta \Psi_{\|}\left(\Delta \Psi_{\perp}\right)^{2}\right\rangle_{c}$. As explained above, in contrast to the standard Eulerian perturbation theory, the resulting power spectrum would remain well behaved at high $k$, with a Zel'dovich-like damping.

An advantage over the Eulerian-based approaches is that we are not sensitive to the "sweeping effect" [2, 3, 24] associated with random advection by long wavelengths of the velocity field, which only move structures by a random uniform shift, because we directly work with relative displacements. As compared with most Eulerian perturbative resummation schemes, this means that we do not need to build a model (from a phenomenological approach or a partial resummation of perturbative diagrams) for different-time Eulerian response functions or propagators, which are governed by the onepoint velocity distribution [4, 30]. This should make such Lagrangian-space approaches more robust because they are not sensitive to such quantities that introduce additional approximations.

\section{Adhesion-like shell-crossing continuation}

Whatever the order up to which standard perturbation theory is exactly recovered by the power spectrum $P^{\|}(k)$, following the procedure described in the previous section, one ingredient is still missing: the nonperturbative shell-crossing regime. In contrast to the standard Eulerian perturbation theory that becomes meaningless after shell crossing (the equations of motion themselves no longer make sense in the multistreaming regime), the Lagrangian approach, which is based on particle trajectories, still makes sense after shell crossing. For instance, in the Zel'dovich approximation, the particles keep following straight lines after shell crossing, $\mathbf{x}(\mathbf{q}, t)=$ $\mathbf{q}+\boldsymbol{\Psi}_{L}(\mathbf{q}, t)$. A similar behavior is implicitly included in the Lagrangian ansatz (35). Shell crossing does not appear in this formalism and particles eventually escape to infinity as in the Zel'dovich approximation: the distributions $\mathcal{P}_{\|}\left(\Delta x_{\|}\right)$and $\mathcal{P}_{\perp}\left(\Delta \mathbf{x}_{\perp}\right)$ widen with time.

This implicitly provides a regularization (or completion) of the Eulerian hydrodynamical equations of motion. Indeed, as noticed above, the latter do not pro- 
vide a complete description of the dynamics because they break down after shell crossing and one should explicitly state how particles behave afterwards. For instance, the Zel'dovich dynamics can also be written in terms of the Eulerian equations of motion [2, 18, 31]. As compared with the gravitational case, one replaces the gravitational potential (which is coupled to the density field through the Poisson equation) with the velocity potential (this is exact at linear order). This yields an Euler equation for the velocity field that decouples from the density field and gives back the trajectories predicted by Lagrangian linear theory. As in the gravitational case, this Euler equation breaks down at shell crossing (when multistreaming appears), and to continue the dynamics at later times one needs to go back to the Lagrangian interpretation in terms of trajectories and state that particles keep moving along their initial direction forever. However, this is not the only possible continuation. For instance, following the "adhesion model" introduced in [18], one can choose to make particles stick together after collisions in order to mimic the trapping in gravitational potential wells. In fact, in dimensions greater than 1, several continuations are possible. In the most convenient geometrical formulation in terms of convex hulls and Legendre transforms, one obtains an intricate process of halo mergings and fragmentations [32, 33], but it is also possible to use another continuation (which has no simple geometrical interpretation), where halos cannot fragment but show complex motions along the shock manifold [34]. These two continuations and the Zel'dovich dynamics are identical before shell crossing and have the same perturbative expansion (they obey the same fluid equations in the single-stream regime); they only differ after shell crossing, and this only appears through nonperturbative terms.

This simple example shows that we could imagine different continuations of the power spectrum (35) into the shell-crossing regime. Thus, Eq. (35) implicitly includes a "Zel'dovich-like" continuation, but one of the main ideas of this paper is that this is not the best choice. Indeed, even though in this section we neglect halo formation, or more precisely the inner halo regions, we would like to obtain a reasonable description of the cosmic web. In terms of the halo model that we use in Sec. IV] below, the one-halo term describes the inner halo shells, and the two-halo term, which is based on the large-scale power spectrum that we consider here, describes the cosmic web with its pancakes and filaments. As is wellknown, such intermediate-scale structures are erased in the Zel'dovich approximation as particles escape to infinity [26]. This has led to the "truncated Zel'dovich approximation" 35, 36], where one removes the initial power at high $k$ to suppress the damping of small-scale structures. More generally, a Zel'dovich-like continuation, such as (35), leads to a falloff of the density power spectrum at high $k$, due to this erasing of small-scale structures, and truncating the initial power cannot provide more than a $k^{-3}$ tail [2, 21, 22] (e.g., for a power-law linear power spectrum $P_{L}(k) \propto k^{n}$ with $-3<n<-1$, we have $P^{\mathrm{Z}}(k) \sim k^{-3+3(n+3) /(n+1)}$ at high $\left.k\right)$.

This means that Zel'dovich-like continuations are not sufficient to describe the cosmic web. Indeed, they miss the formation of pancakes and filaments that give rise to additional power at high $k$. For instance, pancakes, or more precisely, extended sheets of zero thickness, would give a contribution $P(k) \sim k^{-2}$ whereas infinitesimally thin filaments would give $P(k) \sim k^{-1}$. In the actual case of the gravitational dynamics with a hierarchical CDM power spectrum, we do not have such universal and singular structures (pancakes and filaments can show holes of various sizes), and we would rather have a (multi)fractal density field with a typical scaling $P(k) \sim k^{-1.2}$ [associated with a correlation function $\xi(r) \sim r^{-1.8}$ [37 39]]. Another modification is the finite width of the pancakes and filaments, which can be associated with the inner halo structure and is not our concern in this section.

On a quantitative level, as recalled in the Introduction, previous approaches that try to match perturbation theory with a halo model give too little power on intermediate scales, where $\Delta^{2}(k) \sim 1$. This could be traced back to the fact that most resummation schemes converge back to the linear power at high $k$ or even show a sharper cutoff. From the discussion above, one explanation is that to match the very large-scale perturbative regime, where shell crossing is truly negligible, to the high- $k$ regime associated with inner halo regions, we need to take into account intermediate-scale structures such as pancakes that give a non-negligible contribution on transition scales. This means that an "adhesion-like" continuation should be more efficient than the Zel'dovich-like continuation.

As recalled above, in dimensions greater than 1, shell crossing is a difficult problem. In fact, in contrast to the one-dimensional case, defining shell crossing itself is not obvious, as two particles coming from opposite sides may join circular orbits in a potential well without physically crossing each other. In this paper, we take the simple criterion that was used in [19] to evaluate the impact of shell crossing on the power spectrum. If the Lagrangian-space to Eulerian-space mapping is potential, that is, $\mathbf{x}(\mathbf{q}, t)=\partial \Phi / \partial \mathbf{q}$, as in the Zel'dovich and adhesion models, then shell crossing is associated with the loss of convexity of the potential $\Phi(\mathbf{q}, t)$. [In the Zel'dovich dynamics, $\Phi^{Z}$ is of the form $|\mathbf{q}|^{2} / 2+\phi_{L}$, where $\phi_{L}$ is stochastic and given by linear theory, and $\Phi^{\mathrm{Z}}$ loses its initial convexity as $\phi_{L}$ grows with time, while in the adhesion model we take its convex hull, $\operatorname{conv}\left(\Phi^{Z}\right)$, which prevents shell crossing but gives rise to shocks [31 33.] Then, convexity of $\Phi(\mathbf{q}, t)$ implies that $\Delta x_{\|} \geq 0$ (i.e., the longitudinal Eulerian separation does not change sign, for any pair of particles) [40, 41]. Then we can choose $\Delta x_{\|}<0$ as a criterion of shell crossing [19]. This is obvious in one dimension and provides the adequate generalization to higher dimensions for potential mappings. In the case of the gravitational dynamics, the mapping is only potential up to the second order of perturbation theory [42], but we can still expect that it gives a useful 


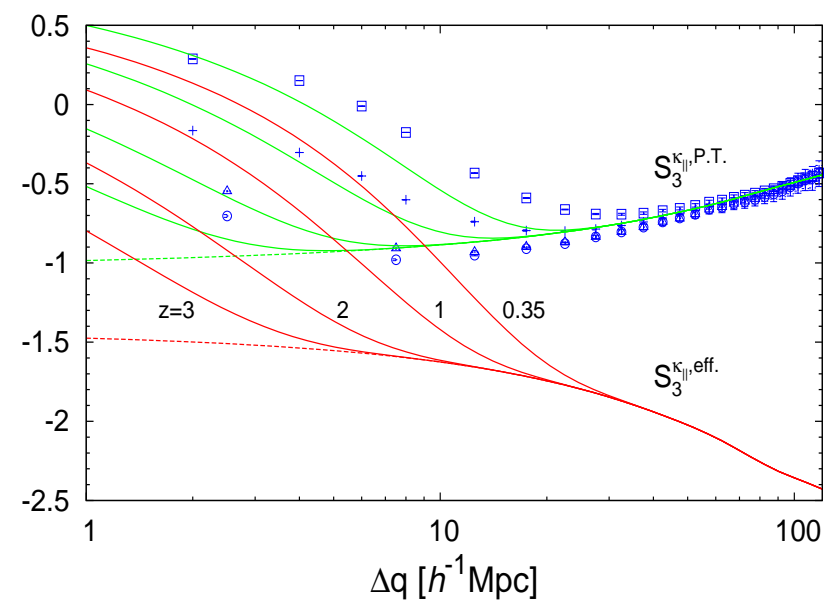

FIG. 6: Skewness $S_{3}^{\kappa} \|$ given by low-order perturbation theory (25) (upper dashed line) and by the effective value (27) (lower dashed line). The solid lines that arise from these two largescale asymptotes are the predictions at redshifts $z=0.35,1,2$ and 3 (from right to left) obtained from the nonperturbative adhesion-like probability distribution (38), determined by the corresponding perturbative part $\mathcal{P}_{\|}$. The points are the results from N-body simulations at $z=0.35$ (squares), $z=1$ (diamonds), $z=2$ (triangles), and $z=3$ (circles).

criterion for the formation of the first large-scale structures.

Therefore, we modify the "perturbative" longitudinal probability distribution (34) by setting $\Delta x_{\|}=0$ to all pairs that had $\Delta x_{\|}<0$. More precisely, we define the "adhesion-like" longitudinal probability distribution as

$$
\mathcal{P}_{\|}^{\text {ad. }}\left(\kappa_{\|}\right)=a_{1} \Theta\left(\kappa_{\|}>0\right) \mathcal{P}_{\|}\left(\kappa_{\|}\right)+a_{0} \delta_{D}\left(\kappa_{\|}\right),
$$

where $\Theta\left(\kappa_{\|}>0\right)$ is the Heaviside function (1 for $\kappa_{\|}>0$ and 0 for $\left.\kappa_{\|}<0\right)$. The parameters $a_{0}$ and $a_{1}$ are set by the constraints $\langle 1\rangle=1$ (i.e., the probability distribution is normalized to unity) and $\left\langle\kappa_{\|}\right\rangle=1$. From the expression (34) we obtain

$$
a_{1}=\left(1+A_{1}\right)^{-1} \text { and } a_{0}=1-a_{1}+a_{1} A_{0},
$$

where we introduce

$$
A_{n}=\int_{0^{+}-\mathrm{i} \infty}^{0^{+}+\mathrm{i} \infty} \frac{\mathrm{d} y}{2 \pi \mathrm{i} \sigma_{\kappa_{\|}}^{2}}\left(\frac{\sigma_{\kappa_{\|}}^{2}}{y}\right)^{n+1} e^{-\varphi_{\|}(y) / \sigma_{\kappa_{\|}}^{2}},
$$

where the contour over $y$ runs to the right of the pole at the origin and to the left of the branch cut at $y_{s}=$ $\alpha-1$. The coefficients $A_{n}$ are nonperturbative and scale as $e^{-(\alpha-1) /\left(\alpha \sigma_{\kappa \|}^{2}\right)}$, which gives

$$
\left(a_{1}-1\right) \sim a_{0} \sim e^{-(\alpha-1) /\left(\alpha \sigma_{\kappa_{\|}}^{2}\right)} .
$$

Therefore, the "perturbative" distribution $\mathcal{P}_{\|}$obtained in Eq. (34) in Sec. IIIB3 and its "adhesion-like" modification (38) are identical to all orders of perturbation theory.
We show in Fig. 6 the skewness $S_{3}^{\kappa \|}$ defined by this new probability distribution (38), using either the perturbative skewness (25) or the effective value (27) for the perturbative part $\mathcal{P}_{\|}$. On large scales, we recover the skewness associated with the regular distribution (34), with a very fast convergence because the deviation decays as $\sim e^{-(\Delta q)^{2} / \sigma_{v}^{2}}$. On small scales, the skewness increases and becomes positive in a fashion similar to the behavior measured in the simulations. As for the negative sign in the perturbative regime that we explained in Sec. IIIB this can be understood from the dynamics. Indeed, because of the "sticking" of particle pairs at $\Delta x_{\|}=0$, in the nonlinear regime (i.e., on small scales), which becomes sensitive to this modification, the low- $\kappa_{\|}$ tail becomes very sharp (it is zero for $\kappa_{\|}<0$ ), whereas the high- $\kappa_{\|}$tail still extends to infinity, albeit with an exponential-like decay. Therefore, the global shape of the probability distribution now shows a broader right tail, in contrast with the perturbative regime shown in Fig. [5 which now leads to a positive skewness. In the actual gravitational case, there is no such exact "sticking" to $\Delta x_{\|}=0$, but there is a trapping within small virialized halos. Thus, collapsed pairs remain bound with a separation set by the typical size $x_{\text {halo }}$ of virialized halos. This plays the role of the left-tail cutoff, which is no longer sharp at $\Delta x_{\|}=0$ but decays on a scale of order $x_{\text {halo }}$, whereas the right tail still extends to infinity and is not strongly affected by smaller-scale virialization (it corresponds to rare voids). Of course, we cannot expect the simple model (38) to provide an accurate prediction for $S_{3}^{\kappa \|}$, even when we use the correct perturbative limit (25) on large scales. However, we can check in Fig. [6 that it already provides a good qualitative description. In particular, it captures the dependence on redshift of the upturn of $S_{3}^{\kappa \|}$, due to these nonperturbative effects that occur after shell crossing.

Then, we modify the perturbative power spectrum (35) by replacing the perturbative probability distribution (34) with its adhesion-like extension (38). Substituting into Eq.(19) gives the "cosmic web" power spectrum

$$
\begin{aligned}
& P_{\text {c.w. }}(k)=\int \frac{\mathrm{d} \Delta \mathbf{q}}{(2 \pi)^{3}} \frac{1}{1+A_{1}}\left\{e^{-\varphi_{\|}\left(-\mathrm{i} k \mu \Delta q \sigma_{\kappa_{\|}}^{2}\right) / \sigma_{\kappa_{\|}}^{2}}+A_{1}\right. \\
& \left.+\int_{0^{+}-\mathrm{i} \infty}^{0^{+}+\mathrm{i} \infty} \frac{\mathrm{d} y}{2 \pi \mathrm{i}} e^{-\varphi_{\|}(y) / \sigma_{\kappa_{\|}}^{2}}\left[\frac{1}{y}-\frac{1}{y+\mathrm{i} k \mu \Delta q \sigma_{\kappa_{\|}}^{2}}\right]\right\} \\
& \times e^{-\frac{1}{2} k^{2}\left(1-\mu^{2}\right) \sigma_{\perp}^{2}},
\end{aligned}
$$

where the contour over $y$ again crosses the real axis between $0<y<\alpha-1$. As in [19], this is a "sticky model" that can be seen as a very simplified version of the full 3D adhesion model. Since we do not modify the transverse motion, the "adhesion" only takes place along the longitudinal direction, which also serves as the signal of shell crossing. Therefore, we only include planar structures (thin pancakes). To describe filaments we should also include some sticking along one transverse direction, but this would require additional ingredients and free pa- 


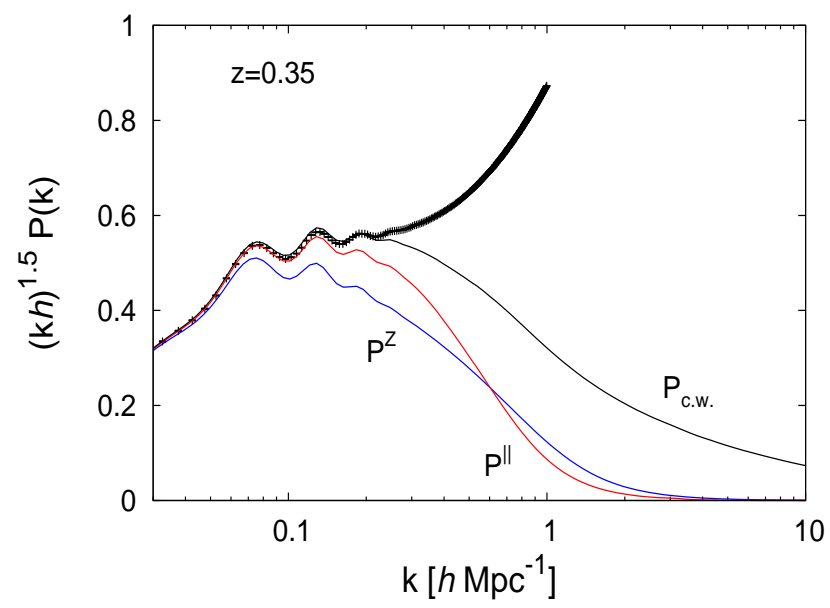

FIG. 7: Matter density power spectrum at $z=0.35$, as in Figs. 1 and 4 We show the results from N-body simulations (data points), the nonlinear Zel'dovich power spectrum $P^{Z}$ (16), the nonlinear ansatz $P^{\|}$(35), and the "adhesion-like" continuation $P_{\text {c.w. }}$ (42) for the "cosmic web".

rameters (e.g., to set the relative mass between filaments and pancakes). Hence, for simplicity we only take into account pancakes as in Eq. (42), which fits in a natural fashion in our framework where we have already kept track of the longitudinal separation.

As explained above, the shell-crossing correction is nonperturbative, and the power spectrum (42) is identical to the power spectrum (35) to all orders over $P_{L}$.

We compare in Fig. 7 the three Lagrangian-space power spectra that we have obtained, the usual Zel'dovich approximation $P^{\mathrm{Z}}$, our nonlinear ansatz $P^{\|}$, and its "adhesion-like" continuation $P_{\text {c.w. }}$. On large scales, the nonperturbative correction is negligible, and we recover the perturbative power spectrum $P^{\|}$, which is somewhat larger than the Zel'dovich power spectrum because of the nonzero skewness $S_{3}^{\kappa \|}$ that allows us to ensure consistency with standard perturbation theory up to one-loop order. On small scales, the nonperturbative correction becomes dominant and gives some extra power, associated with the formation of pancakes, with a high- $k$ tail $\sim k^{-2}$ that decreases more slowly than the Zel'dovich-like tails (that are steeper than $k^{-3}$ ). The nonlinear power spectrum $P_{\text {c.w. }}$ is the Lagrangian ansatz that we use in this paper to describe the "cosmic web".

\section{COMBINING THE "COSMIC WEB POWER SPECTRUM" WITH THE HALO MODEL}

\section{A. The halo model from a Lagrangian point of view}

Because our goal is to build a unified model for the power spectrum that applies from linear to highly nonlin- ear scales, we must combine the perturbative approach described in the previous section (which is systematic and accurate but only applies to large scales) with phenomenological models (that are not systematic and only show an accuracy of $10 \%$ but can be applied to small scales). Following [11, 15], we consider the halo model from a Lagrangian point of view instead of the usual Eulerian framework [4]. This provides a convenient framework to include our Lagrangian perturbative approach within the halo model (the latter being mostly used to describe small, highly nonlinear scales). In particular, the transition from the perturbative large scales, driven by bulk flows, to the inner halo regions, driven by virialization, takes place in a gradual fashion while satisfying matter conservation (i.e., without double counting).

Following [11], we split the average in Eq.(16) over two terms, $P_{1 \mathrm{H}}$ and $P_{2 \mathrm{H}}$, associated with pairs $\left\{\mathbf{q}_{1}, \mathbf{q}_{2}\right\}$ that belong either to a single halo or to two different halos,

$$
P(k)=P_{1 \mathrm{H}}(k)+P_{2 \mathrm{H}}(k),
$$

with

$$
P_{1 \mathrm{H}}(k)=\int \frac{\mathrm{d} \Delta \mathbf{q}}{(2 \pi)^{3}} F_{1 \mathrm{H}}(\Delta q)\left\langle e^{\mathrm{ik} \cdot \Delta \mathbf{x}}-e^{\mathrm{i} \mathbf{k} \cdot \Delta \mathbf{q}}\right\rangle_{1 \mathrm{H}}
$$

and

$$
P_{2 \mathrm{H}}(k)=\int \frac{\mathrm{d} \Delta \mathbf{q}}{(2 \pi)^{3}} F_{2 \mathrm{H}}(\Delta q)\left\langle e^{\mathrm{i} \mathbf{k} \cdot \Delta \mathbf{x}}-e^{\mathrm{ik} \cdot \Delta \mathbf{q}}\right\rangle_{2 \mathrm{H}} .
$$

Here the averages $\langle\ldots\rangle_{1 \mathrm{H}}$ and $\langle\ldots\rangle_{2 \mathrm{H}}$ are the conditional averages, knowing that the pair of length $\Delta q$ belongs to a single halo or to two halos, while $F_{1 \mathrm{H}}$ and $F_{2 \mathrm{H}}$ are the associated probabilities. The approximation of spherical halos in Lagrangian space gives, for the probability that the pair belongs to a single halo [11],

$$
F_{1 \mathrm{H}}(\Delta q)=\int_{\nu_{\Delta q / 2}}^{\infty} \frac{\mathrm{d} \nu}{\nu} f(\nu) \frac{\left(2 q_{M}-\Delta q\right)^{2}\left(4 q_{M}+\Delta q\right)}{16 q_{M}^{3}},
$$

where $q_{M}$ is the Lagrangian radius associated with the mass $M, M=4 \pi \bar{\rho} q_{M}^{3} / 3$, and $f(\nu)$ is the scaling function that defines the halo mass function,

$$
n(M) \frac{\mathrm{d} M}{M}=\frac{\bar{\rho}}{M} f(\nu) \frac{\mathrm{d} \nu}{\nu}, \text { with } \nu=\frac{\delta_{L}}{\sigma(M)} .
$$

Here $\sigma(M)$ is the rms linear density contrast at scale $M$, and $\delta_{L}=\mathcal{F}^{-1}(200)$ is the linear density contrast associated with the nonlinear density threshold that defines collapsed halos, which we choose to equal 200. In numerical computations, we use the fit to the halo mass function from [44], which has been shown to match numerical simulations while obeying the asymptotic large-mass tail $f(\nu) \sim e^{-\nu^{2} / 2}$ [45]. The probability that the pair belongs to two different halos reads as

$$
F_{2 \mathrm{H}}(\Delta q)=1-F_{1 \mathrm{H}}(\Delta q) .
$$

This automatically avoids any double counting in the decomposition (43), and it ensures that we count all matter once. 


\section{B. One-halo term}

The decomposition (43) also corresponds to the onehalo and two-halo terms of the usual halo model [43]. In particular, Eqs.(44) and (46) yield [11, 15]

$$
P_{1 \mathrm{H}}(k)=\int_{0}^{\infty} \frac{\mathrm{d} \nu}{\nu} f(\nu) \frac{M}{\bar{\rho}(2 \pi)^{3}}\left(\tilde{u}_{M}(k)-\tilde{W}\left(k q_{M}\right)\right)^{2} .
$$

Here $\tilde{u}_{M}(k)$ is the normalized Fourier transform of the halo radial profile,

$$
\tilde{u}_{M}(k)=\frac{\int \mathrm{d} \mathbf{x} e^{-\mathrm{i} \mathbf{k} \cdot \mathbf{x}} \rho_{M}(x)}{\int \mathrm{d} \mathbf{x} \rho_{M}(x)}=\frac{1}{M} \int \mathrm{d} \mathbf{x} e^{-\mathrm{i} \mathbf{k} \cdot \mathbf{x}} \rho_{M}(x),
$$

where $\rho_{M}(x)$ is the halo density profile for a halo of mass $M$, and $\tilde{W}(k q)$ is the normalized Fourier transform of the top hat of radius $q$,

$$
\tilde{W}(k q)=\int_{V} \frac{\mathrm{d} \mathbf{q}}{V} e^{\mathrm{i} \mathbf{k} \cdot \mathbf{q}}=3 \frac{\sin (k q)-k q \cos (k q)}{(k q)^{3}} .
$$

To derive Eq. (49) we used the approximation of fully virialized halos: the two particles $\mathbf{q}_{1}$ and $\mathbf{q}_{2}$ have lost all memory of their initial locations and are independently located at random within the halo. As in [11], in numerical computations we use the usual NFW halo profile [46].

The counterterm $\tilde{W}$ in Eq. (49) arises from the counterterm $e^{\mathrm{ik} \cdot \Delta \mathbf{q}}$ of Eq.44) (this subtracts the contribution from the mean density). The computation in [11] would rather give a factor $\left(\tilde{u}_{M}^{2}-\tilde{W}^{2}\right)$, but we prefer to use the factor $\left(\tilde{u}_{M}-\tilde{W}\right)^{2}$ that readily extends to higher-order multispectra as seen in [15]. Moreover, this ensures that the one-halo contribution to the matter power spectrum will decay as $k^{4}$ at low $k$, as implied by the conservation of matter and momentum for small-scale redistributions of matter [47] [whereas the factor $\left(\tilde{u}_{M}^{2}-\tilde{W}^{2}\right)$ only ensures a $k^{2}$ tail, which is consistent with the conservation of matter but not of momentum]. We show in Fig. 22 in the Appendix the impact of this low- $k$ tail of the onehalo term on the power spectrum. We find that using a factor $\left(\tilde{u}_{M}^{2}-\tilde{W}^{2}\right)$, which gives a slower falloff at low $k$, can overestimate the power spectrum by about $5 \%$ on transition scales. Indeed, at higher $k$, in the highly nonlinear regime, the counterterm $\tilde{W}$ is negligible, whereas at lower $k$ the one-halo term itself is negligible. This shows that on transition scales, where $\Delta^{2}(k) \sim 1$, the power spectrum is sensitive to details of the halo model if we require an accuracy of a few percent. Fortunately, this only appears on a limited range of scales and has no impact on the perturbative scales. (However, it is important to ensure that the one-halo term decays at least as $k^{2}$ at low $k$ rather than converging to a constant as in the usual prescription without any counterterm.)

\section{Two-halo term}

At a perturbative level, $F_{1 \mathrm{H}}$ and $P_{1 \mathrm{H}}$ are identically zero while $F_{2 \mathrm{H}}$ is unity, because of the exponential decay of the halo mass function, of the form $e^{-1 / \sigma^{2}(M)}$, in the rare event limit. Then, as noticed in 11], the power spectrum given by perturbation theory is included in the two-halo contribution (45). More precisely, the expansion over powers of $P_{L}$ of $P_{2 \mathrm{H}}$ must recover the standard perturbative expansion. In [11], we used the simple approximation $P_{2 \mathrm{H}}(k) \simeq F_{2 \mathrm{H}}(1 / k) P_{\text {pert }}(k)$, where $P_{\text {pert }}(k)$ is the power spectrum given by the Eulerian "steepestdescent" resummation scheme developed in 67, 24]. Any other Eulerian or Lagrangian resummation scheme could be used, provided it is well behaved at high $k$, where it becomes subdominant with respect to the one-halo term. (This excludes the standard perturbation theory, which grows too fast at high $k$, unless one adds an extra high- $k$ cutoff.)

However, the approaches investigated in [1] did not manage to provide a fully satisfactory matching to the highly nonlinear regime, because they predicted too little power on intermediate scales (where $\Delta^{2}(k) \sim 1-10$ ). This can be traced to the fact that they usually go back to the linear power at most at high $k$, which leads to insufficient power on transition scales where $P_{2 \mathrm{H}}$ and $P_{1 \mathrm{H}}$ are of the same order. Another problem was that Lagrangian perturbative schemes, which would be more convenient to embed within Eq.45) and to extend to redshift space, made this lack of power even worse, because they usually display a strong cutoff at high $k$. In this paper, our goal is to improve over [11] by implementing a Lagrangian perturbative scheme that is free of this problem and provides reasonably accurate predictions up to the transition scales. Thus, we use the "cosmic web" power spectrum developed in Sec. III as a basis of our two-halo term, which we write as

$$
\begin{gathered}
P_{2 \mathrm{H}}(k)=\int \frac{\mathrm{d} \Delta \mathbf{q}}{(2 \pi)^{3}} F_{2 \mathrm{H}}(\Delta q)\left\langle e^{\mathrm{i} \mathbf{k} \cdot \Delta \mathbf{x}}\right\rangle_{\Delta q}^{\mathrm{vir}} \frac{1}{1+A_{1}} \\
\times e^{-\frac{1}{2} k^{2}\left(1-\mu^{2}\right) \sigma_{\perp}^{2}}\left\{e^{-\varphi_{\|}\left(-\mathrm{i} k \mu \Delta q \sigma_{\kappa_{\|}}^{2}\right) / \sigma_{\kappa_{\|}}^{2}}+A_{1}\right. \\
\left.+\int_{0^{+}-\mathrm{i} \infty}^{0^{+}+\mathrm{i} \infty} \frac{\mathrm{d} y}{2 \pi \mathrm{i}} e^{-\varphi_{\|}(y) / \sigma_{\kappa_{\|}}^{2}}\left(\frac{1}{y}-\frac{1}{y+\mathrm{i} k \mu \Delta q \sigma_{\kappa_{\|}}^{2}}\right)\right\} .
\end{gathered}
$$

We recognize the cosmic web power spectrum given by Eq.421, to which we added the factors $F_{2 \mathrm{H}}$ and $\left\langle e^{\mathrm{ik} \cdot \Delta \mathbf{x}}\right\rangle_{\Delta q}^{\operatorname{vir}}$. As explained in Eqs. (43)-(45), the factor $F_{2 \mathrm{H}}$, given by Eqs. (48) and (46), ensures that mass is conserved: there is no double counting of particle pairs. The factor $\left\langle e^{\mathrm{i} \mathbf{k} \cdot \Delta \mathbf{x}}\right\rangle_{\Delta q}^{\text {vir }}$ is associated with small-scale motions.

Indeed, as seen from the derivation of Eq.(42) in Sec. III the cosmic web power spectrum $P_{\text {c.w. }}$ arises from the statistical average of $e^{\mathrm{i} \mathbf{k} \cdot \Delta \mathbf{x}}$ due to large-scale motions, associated with the bulk flows described by perturbation theory that we regularize by an adhesion-like 
continuation. As noticed in Sec. IIIC this simple procedure takes into account the formation of idealized, infinitesimally thin pancakes. In the actual gravitational process, pancakes and filaments have a finite width, as particles keep moving for some time after shell crossing instead of instantaneously sticking together, and have finite-size turnaround radii and virialized orbits. More generally, we can split the motion of particles into two components, a first one associated with large-scale bulk flows, which was considered in Sec. III and corresponds to the "skeleton" of the large-scale structures, and a second one associated with small-scale virialized motions, which gives some thickness to this skeleton. In particular, for hierarchical linear power spectra with a high- $k$ tail that does not decrease faster than $k^{-3}$, all particles are expected to belong to collapsed objects (this may not be the case for linear power spectra with less power at high $k$ ). Then, making the approximation that these small-scale and large-scale motions are decorrelated, we write

$$
\left\langle e^{\mathrm{i} \mathbf{k} \cdot \Delta \mathbf{x}}\right\rangle=\left\langle e^{\mathrm{ik} \cdot \Delta \mathbf{x}}\right\rangle^{\text {bulk }}\left\langle e^{\mathrm{i} \mathbf{k} \cdot \Delta \mathbf{x}}\right\rangle^{\text {vir }}
$$

The first part was the focus of Sec. III and corresponds to the cosmic web power spectrum $P_{\text {c.w. }}$. Assuming, as in the derivation of the one-halo term (49), full virialization [11], that is, that the two particles $\mathbf{q}_{1}$ and $\mathbf{q}_{2}$ are independently located at random in their host halo, we write

$$
\begin{aligned}
\left\langle e^{\mathrm{i} \mathbf{k} \cdot \Delta \mathbf{x}}\right\rangle_{\Delta q}^{\operatorname{vir}} & =\frac{\int \frac{\mathrm{d} \nu_{1}}{\nu_{1}} f\left(\nu_{1}\right)\left\langle e^{\mathrm{ik} \cdot \mathbf{x}_{1}}\right\rangle_{M_{1}}}{\int \frac{\mathrm{d} \nu_{1}}{\nu_{1}} f\left(\nu_{1}\right)} \frac{\int \frac{\mathrm{d} \nu_{2}}{\nu_{2}} f\left(\nu_{2}\right)\left\langle e^{\mathrm{ik} \cdot \mathbf{x}_{2}}\right\rangle_{M_{2}}}{\int \frac{\mathrm{d} \nu_{2}}{\nu_{2}} f\left(\nu_{2}\right)} \\
& =\left[\frac{\int_{0}^{\nu_{\Delta q / 2}} \frac{\mathrm{d} \nu}{\nu} f(\nu) \tilde{u}_{M}(k)}{\int_{0}^{\nu_{\Delta q / 2}} \frac{\mathrm{d} \nu}{\nu} f(\nu)}\right]^{2}
\end{aligned}
$$

In contrast with the one-halo case (49) where the two particles belong to the same halo, which gave rise to the factor $\tilde{u}_{M}^{2}$, here the two particles belong to two different halos, whence the integration over two halo mass functions, each one with its factor $\tilde{u}_{M}$. In Eq.(54), we only integrate over halos of radius smaller than $\Delta q / 2$, to take into account in an approximate fashion that if a pair of separation $\Delta q$ belongs to two different halos, these halos are unlikely to have a radius much greater than $\Delta q / 2$. Thus, massive halos only contribute to the one-halo term (49) and to the two-halo term (52) at large distances.

We show in Fig. 22 in the Appendix the impact of this "virial damping" factor on the matter power spectrum, by plotting the deviation that would be obtained by neglecting this term. Because of the upper bound on halo mass in Eq. (54), this factor does not induce a significant damping of the power spectrum (52) on large scales. Indeed, in the weakly nonlinear regime where the twohalo term is dominant, power at wavenumber $k$ typically comes from pairs of initial separation $\Delta q \sim 1 / k$, which selects halo radii $q_{M}$ that are smaller than $1 / k$ for which the factor $\tilde{u}_{M}(k)$ is close to unity. On small, highly nonlinear scales it yields a greater damping as $1 / k$ becomes smaller than the typical size of the halos. However, on these scales, the matter power spectrum given by our approach is not accurate to better than $10 \%$ because of the one-halo term itself, which involves the halo profiles and their concentration parameters that are not modeled to a very high accuracy. Nevertheless, we include this factor in our computations because it naturally arises in our framework.

\section{Nonperturbative effects on large scales}

Thus, in our approach we include nonperturbative and shell-crossing effects on the large-scale power spectrum through the factors $F_{2 \mathrm{H}}$ and $\left\langle e^{\mathrm{i} \mathbf{k} \cdot \Delta \mathbf{x}}\right\rangle_{\Delta q}^{\mathrm{vir}}$, associated with halo formation and virialized motions, and through the adhesion-like continuation described in Sec. IIIC (in addition to the one-halo term itself). This is different from recent Eulerian-space works [48, 49] that propose to include the impact of such nonperturbative effects on large scales through additional terms in the hydrodynamical equations of motion of the fluid approximation, that may be obtained by a combination of coarse-graining (that sets the form of these terms) and phenomenology (they include coefficients that are measured in simulations). Indeed, in our approach the hydrodynamical equations of motion enter through the perturbative expansion that they imply for the power spectrum, which is not affected by these nonperturbative effects. Then, the impact of a small-scale velocity dispersion, due to virialized motions, is described by the factor $\left\langle e^{\mathrm{ik} \cdot \Delta \mathbf{x}}\right\rangle_{\Delta q}^{\mathrm{vir}}$ in Eq.(53). This would play the role of some pressurelike terms included in [48, 49]. The trapping within potential wells, that is described in a simplified manner by the nonperturbative correction (42) associated with the adhesion-like continuation, may also be described within such methods through a pressure or viscosity term, as in the original adhesion model [18], where the pressureless Euler equation is replaced with the Burgers equation.

As seen in Sec. IIIC, the "sticky model" that we use for the cosmic web power spectrum corresponds to a onedimensional adhesion model in the inviscid limit, that is; when the viscosity parameter $\nu$ is sent to $0^{+}$. The associated Euler equation is also known as the Burgers equation 50], introduced as a model for turbulence. Within a specific geometrical formulation of the inviscid limit, the equations of motion can be explicitly solved in any dimension, through Legendre transforms and convex hulls [31, 33]. However, it remains very difficult to obtain explicit results for the statistical properties of the associated density and velocity fields, except for a few cases in one dimension (for power-law initial conditions with $n=-2$ [51, 52] or $n=0$ [53, 54]). If we keep a finite viscosity parameter $\nu$, the solution is more regular (there are no shocks), but there are no explicit results for statistical properties of the displacement field either. This is why we use the simple model of Sec. IIIC, which provides the simple explicit expression (42), whereas a fully three- 
dimensional adhesion model with finite viscosity would yield more intricate path integrals. A second reason for our choice is to avoid introducing additional free parameters. Indeed, by introducing a finite viscosity parameter, which may also depend on the local density and on time, as well as other nonlinear terms in the equations of motion, one needs to build a model for these new parameters or functions. Although these parameters may be estimated from simulations, we prefer in this paper to stick to the simplest possible modeling that captures some of the nonperturbative shell-crossing effects. It may be possible to improve over this first step by considering more realistic equations of motion (e.g., see [55, 56] for earlier works), but we leave this for future studies.

Another approach to take into account such effects would be to go back to the Vlasov equation of motion 5759]. In principle, this could provide systematic schemes, but the methods that have been proposed so far lead to heavier computations, and no fast and accurate method has been presented yet.

As noticed in the Introduction, the advantage of the Lagrangian-space framework over the Eulerian-space framework is that particle trajectories can describe both the single-stream and multistream regimes, which allows us to include these nonperturbative effects in the simple fashion described in the previous sections. However, this approach is not fully systematic, and it involves some phenomenological insight.

\section{NUMERICAL RESULTS}

\section{A. WMAP5 cosmology}

We first compare our model with numerical simulations for a WMAP5 cosmology, that is, with the set of cosmological parameters $h=0.701, \Omega_{\mathrm{m}}=0.279, \sigma_{8}=0.8159$, $n_{s}=0.96$, and $w_{\mathrm{de}}=-1$. While we use the N-body data of [14] on large scales $\left(k<0.3 h \mathrm{Mpc}^{-1}\right)$, we use the measurements of [11] on smaller scales. This is because the former one is tuned to give an accurate power spectrum on BAO scales and has a larger total volume $\left[60 \times\left(2048 h^{-1} \mathrm{Mpc}\right)^{3} \sim 515 h^{-3} \mathrm{Gpc}^{3}\right]$, while the latter gives more reliable results over a wide range in $k$ by carefully combining the measurements from some simulations with different resolutions the highest-resolution simulation employs $2048^{3}$ particles in a $\left(512 h^{-1} \mathrm{Mpc}\right)^{3}$ cube]. Similarly, we use the simulation results from these papers for the two-point correlation function: data points from [14] are shown on BAO scales (from 70 to $140 h^{-1} \mathrm{Mpc}$ ), while those from [11] are depicted on smaller separations.

We show our results for the redshifts $z=3,1$, and 0.35 , which was already used in the previous sections to illustrate the building blocks of our model. The redshift $z=0.35$ is the lowest available redshift for this set of simulations (but we also consider $z=0$ in another set of simulations in Sec. VB 1). However, it is also relevant from an observational point of view, as recent or upcoming redshift surveys aiming at measuring cosmic expansion or structure growth via baryon acoustic oscillations and redshift-space distortions probe similar redshifts (e.g., the SDSS LRG sample 60, 61]) or higher redshifts (e.g., $z \sim 0.57$ for BOSS DR9 62]).

\section{Power spectrum}

We show in Fig. 8 the final power spectrum obtained by our model, combining the cosmic web power spectrum obtained in Sec. III with the halo model. We clearly see how the two-halo and one-halo contributions are dominant on large and small scales, respectively. The transition takes place around $k \sim 1 h \mathrm{Mpc}^{-1}$ at low redshift, in a gradual manner. In contrast with previous studies [11, 15], we no longer underestimate the power spectrum on these transition scales, and we obtain a good agreement up to the highly nonlinear regime. This is because our two-halo contribution is no longer based on a perturbative power spectrum that goes back to the linear power at high $k$ but on the "cosmic web" power spectrum of Sec. III, which shows more power on nonlinear scales because it takes into account intermediate-scale structures such as pancakes. This gives a more realistic basis that appears indeed to provide a better match to the N-body results.

Although our simple modeling takes into account the formation of pancakes, as described in Sec. III it does not include filaments. Indeed, this would require going beyond the linear regime in more than one dimension, to model the trapping of particles within filaments. Then, we may expect an underestimation of the power spectrum on transition scales. We do not try to remedy for this effect in this paper, and Fig. 8 shows that the impact on our results is not very large. In fact, it is likely that this loss of power is hidden within the limited accuracy of our model (due, for instance, to the ambiguities associated with the splitting over one-halo and two-halo terms, while the actual cosmic web is clearly more complex, as pancakes, filaments, and virialized objects are connected with each other without sharp symmetric boundaries.).

The upper row in Fig. 8 clearly shows how the slope of the power spectrum evolves with redshift. For CDM power spectra, the transition scale shifts to higher $k$ at higher redshift, which leads to a "redder" power spectrum (the local slope $n$ of the linear power spectrum decreases and typically shifts from -1.5 to -2.5$)$. In turn, this yields a two-halo contribution that becomes larger with respect to the one-halo contribution on highly nonlinear scales. In retrospect, this explains why the underestimation of the power spectrum on transition scales was more severe at higher redshift in [11, 15]. Although using the "cosmic web" power spectrum of Sec. III is a significant improvement over these previous works, our two-halo contribution is unlikely to be very accurate at high $k$ and this is probably one of the reasons for the discrepancy found in the rightmost panels of Fig. 8 at 

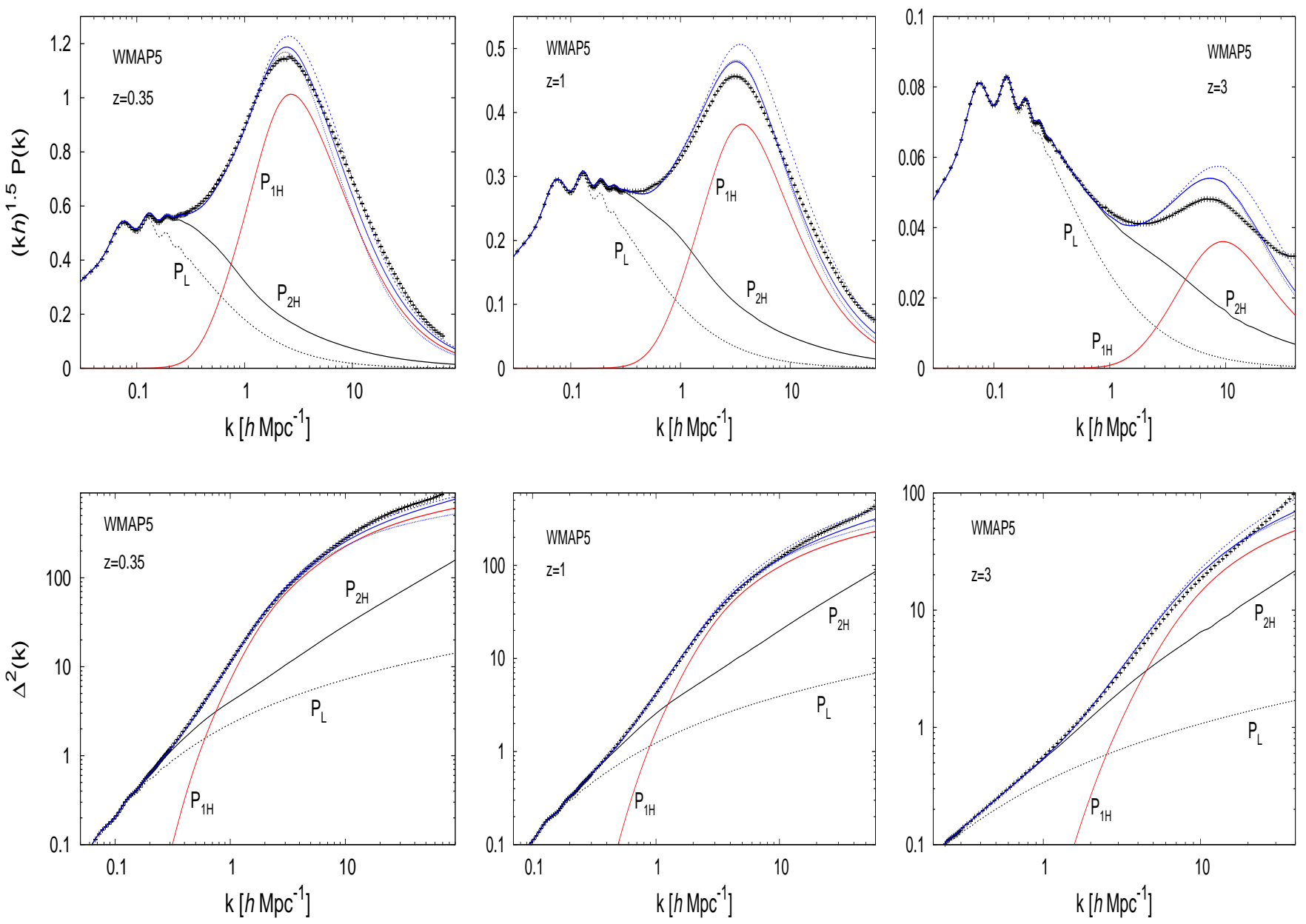

FIG. 8: Upper row: matter density power spectrum $P(k)$ at $z=0.35,1$, and 3 , from left to right, multiplied by a factor $(k h)^{1.5}$ as in Fig. 17. Lower row: logarithmic matter power spectrum, $\Delta^{2}(k)=4 \pi k^{3} P(k)$. We show the linear power spectrum, the twohalo and one-halo contributions $P_{2 \mathrm{H}}(52)$ and $P_{1 \mathrm{H}}$ (49), the full nonlinear power spectrum (43), and the results from numerical simulations. For the full nonlinear power spectrum (43), in addition to the result obtained with the mass-concentration relation [55) (solid line) we also show the results obtained using the mass-concentration relations from [63] and [64] (dashed lines).

$k>3 h \mathrm{Mpc}^{-1}$. On these small scales, we may overestimate the two-halo contribution.

Another source of inaccuracy in this regime is the uncertainty of the halo-profile parameters themselves, in particular the mass-concentration relation $c(M)$. We show in Fig. 8 the results obtained by using for $c(M)$ the fits to N-body simulations from [63] and [64], as well as the formula

$$
c(M)=11\left(\frac{M}{2 \times 10^{12} h^{-1} M_{\odot}}\right)^{-0.1}(1+z)^{-1.5},
$$

for $0.35 \leq z \leq 3$, where halos are defined by a density contrast of 200 with respect to the mean density of the Universe. This gives a power spectrum that is in between those obtained with the fits from [63] and [64] and gives a slightly better match to our simulations at high $k$. In principle, the relation $c(M)$ used for the power spectrum is expected to be slightly different from the one measured in simulations because of its finite scatter, as inclusion in the matter power spectrum is associated with an implicit weight that may differ from the averaging procedure used to measure the relation $c(M)$ in the simulations. In any case, we can see in Fig. 8 that the discrepancy between our model and the power spectrum measured in the N-body simulation is of the same order as the difference between the predictions that use either [63] or 64] for $c(M)$. This means that the power spectrum at these high wave numbers still shows an uncertainty on the order of $10 \%$, whether it is computed through the halo model or directly from the N-body simulations. In particular, shot noise certainly explains part of the rise of the power spectrum measured in the $\mathrm{N}$ body simulations at $z=3$ for $k>20 h \mathrm{Mpc}^{-1}$ above our predictions (lower-right panel in Fig. 8). This is clearly 


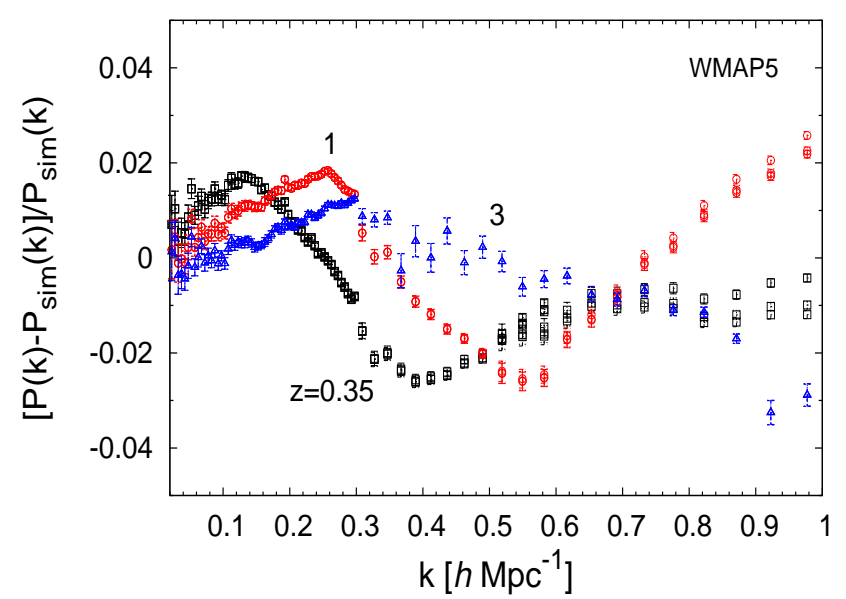

FIG. 9: Relative deviation between the density power spectrum predicted by our model and the result from numerical simulations, at $z=0.35$ (black squares), 1 (red circles), and 3 (blue triangles). We show the results (which almost coincide) obtained using the mass-concentration relation (55) and the ones from [63] and 64].

seen from the comparison with the panels in the lower row obtained at lower redshift, which probe deeper into the nonlinear regime before being affected by shot noise, where we can see that the logarithmic power spectrum follows the shape predicted from the halo model. In particular, at $z=0.35$ we clearly see the slowing down of the growth of $\Delta^{2}(k)=4 \pi k^{3} P(k)$ in the highly nonlinear regime, and we would expect a similar behavior at $z=3$. (Actually, we would expect a slightly faster slowdown because the local slope $n$ of the linear power spectrum is redder.) Therefore, in this regime it seems that semianalytical approaches like ours, based on the halo model, are competitive with direct N-body simulations. (At very high $k$, the semianalytic approaches are expected to remain reasonable, because they are based on a physically reasonable ansatz and/or assumption, whereas the direct results from simulations suffer from shot noise.)

We show in Figs. 9 and 10 the relative deviation between the power spectrum predicted by our model and the N-body measurements. We obtain an accuracy of about $2 \%$ up to $k \sim 0.3 h \mathrm{Mpc}^{-1}$, and $5 \%$ up to $k \sim 3 h \mathrm{Mpc}^{-1}$, for $z \geq 0.35$. The small underestimation of the power spectrum on the transition scales $\left(k \sim 0.5 h \mathrm{Mpc}^{-1}\right.$ at $\left.z \leq 1\right)$ may be due to the fact that filaments are not explicitly included in our model of the cosmic web. The accuracy degrades rapidly in the highly nonlinear regime, because of the uncertainty of the halo model and of the N-body simulations themselves (in particular because of shot noise at very high $k$ ). Fortunately, as shown in Fig. 9, the uncertainties of the halo model (i.e., the parameters of halo profiles) do not contaminate the predictions for the power spectrum on large scales, $k<1 h \mathrm{Mpc}^{-1}$. Therefore, these large scales remain a ro-

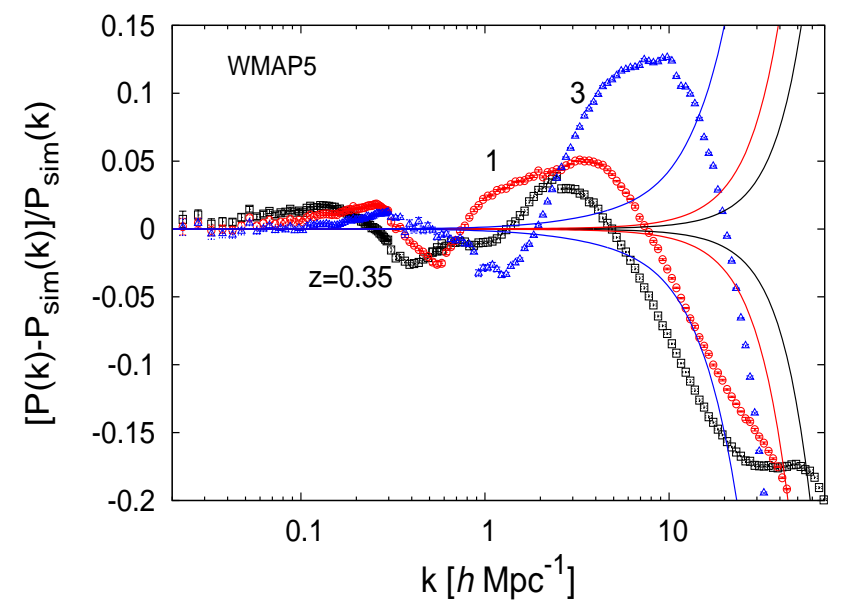

FIG. 10: Relative deviation between the density power spectrum predicted by our model and the result from numerical simulations, at $z=0.35$ (squares), 1 (circles), and 3 (triangles). The solid lines are estimates of the effect of the shot noise in numerical simulations (smaller impact at lower redshift).

bust probe of cosmology. This is also one interest of such analytical approaches that are complementary to numerical simulations: they allow us to estimate the impact of different processes on the final power spectrum and to estimate the range of wave numbers that are not affected by small-scale uncertainties and can be safely used to constrain cosmology up to a good accuracy.

\section{Two-point correlation function}

We show in Fig. 11] our results for the matter density two-point correlation $\xi(x)$, given by

$$
\xi(x)=4 \pi \int_{0}^{\infty} \mathrm{d} k k^{2} P(k) \frac{\sin (k x)}{k x} .
$$

As in previous works [11, 15, 65, 66], we can see that using a well-behaved perturbative contribution that includes one-loop contributions provides a good accuracy on baryon acoustic oscillation (BAO) scales. In particular, we reproduce the well-known damping of the baryonic peak at $\sim 105 h^{-1} \mathrm{Mpc}$ as compared with linear theory. We can see in the lower panel the transition between the two-halo and one-halo contributions, at $x \sim 1 h^{-1} \mathrm{Mpc}$. As for the power spectrum, we obtain a significant improvement over previous studies [11, 15], as we no longer underestimate the two-point correlation on these transition scales. In particular, we recover the shape of the two-point correlation from linear to highly nonlinear scales. Again, the two-halo contribution becomes significantly greater than the linear correlation (or the linear power spectrum in Fig. 8) on small scales 

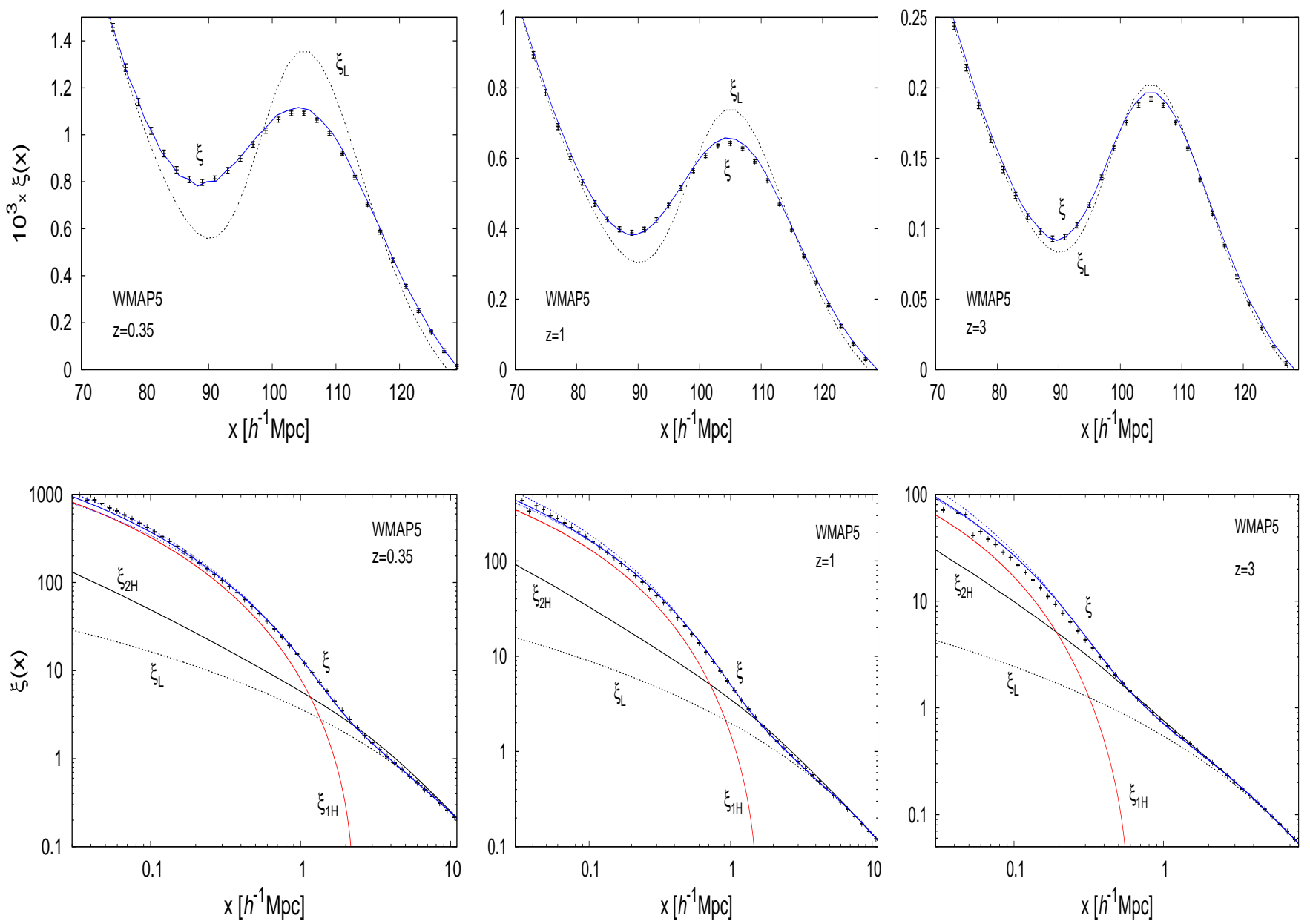

FIG. 11: Upper row: matter density correlation function $\xi(x)$ at $z=0.35,1$, and 3 , from left to right, multiplied by a factor $10^{3}$, on BAO scales. Lower row: correlation function $\xi(x)$ on smaller scales. We show the linear correlation $\xi_{L}$, the two-halo and one-halo contributions $\xi_{2 \mathrm{H}}$ and $\xi_{1 \mathrm{H}}$ from Eqs.(52) and (49), and the full nonlinear correlation $\xi$ from Eq.(43) (using the mass-concentration relation [55) (solid line) and the fits from [63] and 64] (dashed lines)). The points are the results from numerical simulations.

because of the adhesion-like continuation explained in Sec. IIIC] This is more important at higher redshift because the local slope of the linear power spectrum on transition scales becomes redder while our "cosmic web" power spectrum shows a universal $k^{-2}$ tail. The good match with simulations on transition scales suggests that using such a two-halo contribution that is greater than the linear one on nonlinear scales is an important ingredient for recovering the full nonlinear power spectrum.

On small scales, the discrepancy between our model and the numerical simulations is again on the order of the scatter due to the uncertainty of the halo-model massconcentration relation. Fortunately, this only affects very small scales, where the physics of baryons should also be included (for instance, through the halo-model parameters) 67].

We show in Figs. 12 and 13 the relative deviation between the two-point correlation predicted by our model and the one measured in the N-body simulations. We obtain an accuracy of about $2 \%$ on the BAO scales and of about $5 \%$ down to $0.5 h^{-1} \mathrm{Mpc}$. Again, the accuracy degrades on smaller scales because of the uncertainties of the halo-model parameters and of the finite resolution of the N-body simulations themselves. On very large scales, the statistical error bars of the simulations grow because of the finite box size and become much larger than the inaccuracy of the analytical model. In fact, it seems that we predict more power than is measured in the simulations by about $2 \%$ on large scales. Part of this offset may be due to a lack of large-scale power in the N-body simulations because of their finite size. Indeed, we obtain a better agreement on intermediate scales, $20<x<60 h^{-1}$ Mpc. Moreover, the twopoint correlation function changes sign and vanishes at $x_{0} \sim 130 h^{-1} \mathrm{Mpc}$, so that small absolute deviations are amplified after we take the ratio to $\xi_{\operatorname{sim}}(x)$, and the rela- 


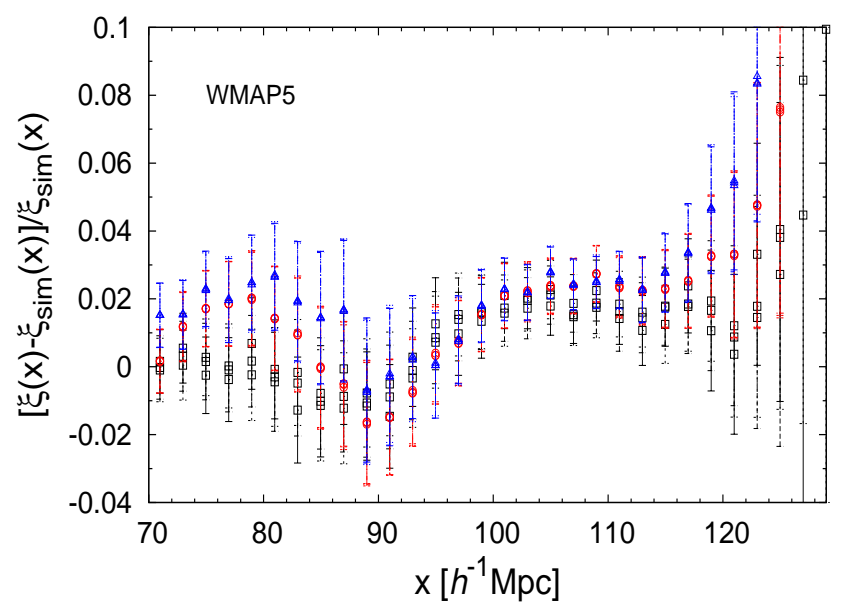

FIG. 12: Relative deviation between the two-point correlation $\xi(x)$ predicted by our model and the result from numerical simulations, at $z=0.35$ (squares), 1 (circles), and 3 (triangles). We show the results (which almost coincide) obtained using the mass-concentration relation (55) and the ones from [63] and [64]. The error bars are the statistical error bars of the N-body simulations.

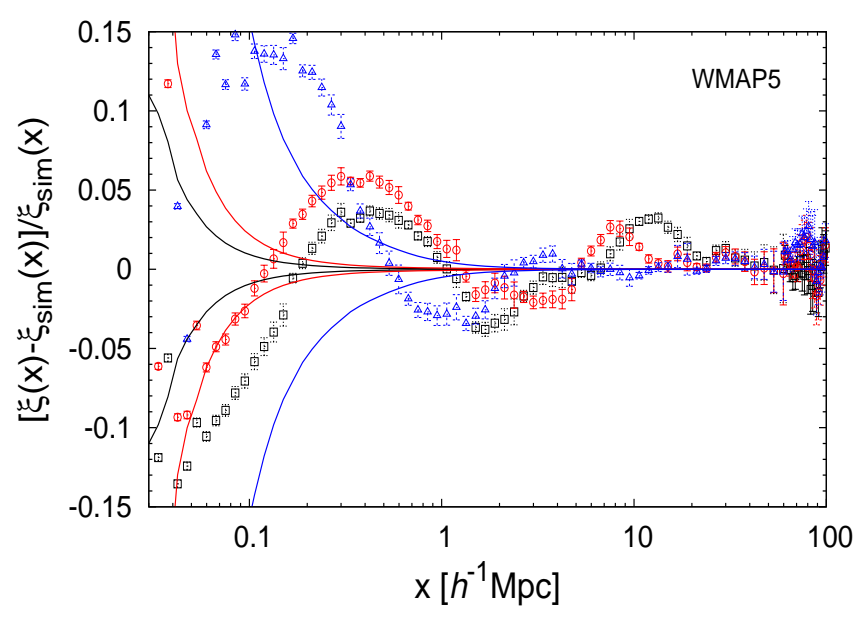

FIG. 13: Relative deviation between the two-point correlation $\xi(x)$ predicted by our model and the result from numerical simulations, at $z=0.35$ (squares), 1 (circles), and 3 (triangles). The error bars are the statistical error bars of the N-body simulations. The solid lines are estimates of the effect of the shot noise in numerical simulations (smaller impact at lower redshift).

tive deviation becomes infinite at $x_{0}$ because of the finite accuracy of models and simulations. Thus, as for the power spectrum, semianalytical approaches like ours appear competitive with direct N-body simulations.

In this paper, we have not considered the impact of baryon physics on the matter density power spectrum

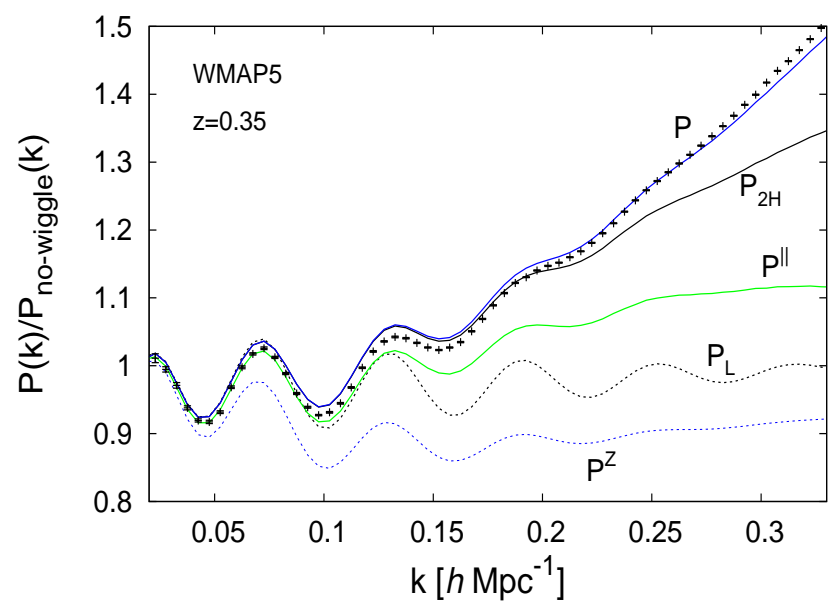

FIG. 14: Ratio of the power spectrum $P(k)$ to a reference linear power spectrum $P_{\text {no-wiggle }}(k)$ without baryon oscillations, at $z=0.35$ We show the WMAP5 linear power $P_{L}$, the Zel'dovich power spectrum $P^{Z}$ from Eq.(16), the nonGaussian perturbative power spectrum $P^{\|}$from Eq. (35), the two-halo contribution $P_{2 \mathrm{H}}$, and the full nonlinear power spectrum $P(k)$. The points are the results from numerical simulations.

and correlation function. Hydrodynamic simulations that include a strong AGN feedback (to cure the overcooling problem and to reproduce X-ray data) suggest that such effects can modify the power spectrum by $10 \%$ at $k \sim 1 h \mathrm{Mpc}^{-1}$ at $z=0$ 68. Fortunately, most of these effects may be described through modifications to the halo model [69] (e.g., by distinguishing the stellar, gas, and dark matter profiles around and within virialized halos). This may also be incorporated within our framework. In this respect, semianalytic approaches like ours can be used to investigate the impact of such modifications of the halo model parameters onto integrated quantities, such as the matter power spectrum or correlation function, and in particular how they affect different scales.

\section{Baryon acoustic oscillations}

In this section, we focus on BAO scales to investigate how the oscillations in $P(k)$ and the peak in $\xi(r)$ depend on the details of the model. As usual, to emphasize the baryon acoustic oscillations, we show in Fig. 14 the ratio of the nonlinear power spectrum $P(k)$ to a reference reference linear power spectrum $P_{\text {no-wiggle }}(k)$ without baryon oscillations, at $z=0.35$. (This is the lowest redshift of this set of simulations, but it also corresponds to the range probed by some surveys that use the baryon oscillations to probe cosmology [60, 61].) Because of high-order mode couplings, all nonlinear results plotted in Fig. 14 show a damping of high- $k$ oscillations, as 


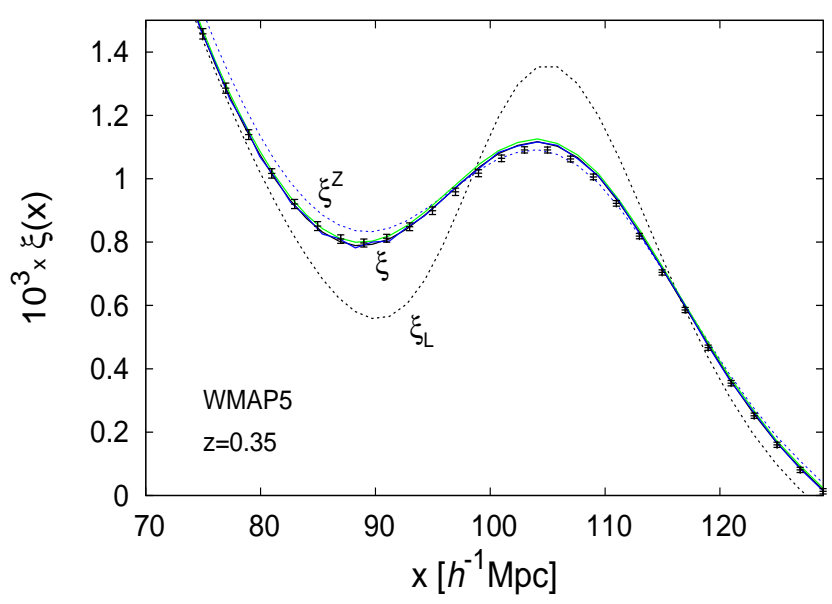

FIG. 15: The matter density correlation function $\xi(x)$ at $z=0.35$, multiplied by a factor $10^{3}$, on BAO scales. The points are the results from numerical simulations. We show the linear correlation $\xi_{L}$ (black dotted line), the correlation $\xi^{Z}$ associated with the Zel'dovich power spectrum (blue dotted line that is slightly above the simulation results at $x \sim 85 h^{-1} \mathrm{Mpc}$ ), and the correlations $\xi^{\|}, \xi_{2 \mathrm{H}}$, and $\xi$, which almost coincide with each other and closely follow the simulations.

compared with the linear power spectrum, where we can still distinguish the oscillations at $k \sim 0.25 h \mathrm{Mpc}^{-1}$ and $k \sim 0.31 h \mathrm{Mpc}^{-1}$. This damping is common to most perturbative schemes that go beyond linear order, because the contribution to the full nonlinear power spectrum of the linear part decreases (e.g., because it is multiplied by decaying propagators [65]), whereas the higher-order contributions mix different wave numbers (through highorder convolutions of the linear power) and smooth the final power.

From a physical point of view, this damping is due to the displacements of matter particles, through largescale bulk flows and small-scale virial motions [70]. This broadens the peak in the real-space correlation function (as seen in Fig. 15), over a width of a few Mpc set by the typical relative displacements of particle pairs. Then, this yields a damping of the oscillations in Fourier space. As shown by Fig. 14, this effect is already captured by the Zel'dovich power spectrum $P^{Z}$ (16), which corresponds to a Gaussian approximation for particle displacements. However, in agreement with the analysis in Sec. IIIA. this also yields an overall damping of the power spectrum that is too strong. Then, the perturbative approximation $P^{\|}$(35) increases the power by going beyond the Gaussian approximation (taking into account the exact one-loop contribution through the skewness of the relative displacement, as in Fig. (5), and the nonperturbative adhesion-like continuation of Sec. IIIC, which enters the two-halo term $P_{2 \mathrm{H}}$ (52), provides a further increase (associated with pancakes). These two nonlinear correc- tions, which involve high-order mode couplings, do not keep much of the initial linear oscillations, and this even further damps the relative importance of the oscillations at high $k$. Finally, the one-halo contribution, which corresponds to the difference $P(k)-P_{2 \mathrm{H}}(k)$, only becomes important for $k>0.23 \mathrm{hMpc}^{-1}$ and is also very smooth.

We show the real-space correlation function associated with these different models in Fig. 15. We clearly see how the damping of the oscillations found in Fig. 14 corresponds to a broadening of the real-space peak, for all nonlinear models. One striking result is that all nonlinear correlations are very close to each other, despite the differences seen in Fig. 14. In particular, $\xi^{\|}, \xi_{2 \mathrm{H}}$, and $\xi$ cannot be distinguished in this figure. This shows that small-scale virial motions are largely subdominant as compared with large-scale bulk flows, with respect to the broadening of the acoustic peak. Moreover, the Zel'dovich approximation $\xi^{\mathrm{Z}}$ already provides a remarkably good description of the broadening, even though it shows a small but noticeable departure from the simulations. (Of course, these curves start to show significant deviations from each other on smaller nonlinear scales, $x<4 h^{-1} \mathrm{Mpc}$.) This shows that even though the baryon acoustic peak is significantly modified from linear theory, all reasonable Lagrangian-space-based models are able to model this nonlinear evolution (see also [16, 29]). This also holds for the simplest one, i.e. the Zel'dovich approximation, although it shows small departures from simulations, and higher-order models (i.e., that include one-loop corrections) provide a very good match. This explains why reconstruction techniques [71], which are inspired by the Zel'dovich approximation, perform very well. For observational purposes, this confirms again that the baryon acoustic peak is a very robust probe of cosmology [70], and that the real-space correlation may be more convenient than the Fourier-space power spectrum, because these nonlinearities may be more naturally understood as particle displacements than resonant wave couplings [2, 4].

Redshift-space distortions also have a (smaller) impact on the acoustic peak of the correlation function [71], but we leave the study of these effects for future works.

\section{B. Other cosmologies}

To further test our model, we also compare our predictions with numerical simulations for two other cosmologies.

\section{WMAP3 cosmology}

We first use an older set of WMAP3 simulations, with $h=0.734, \Omega_{\mathrm{m}}=0.234, \sigma_{8}=0.76, n_{s}=0.961$, and $w_{\mathrm{de}}=-1$, performed in [72]. These simulations have a smaller box size $\left(1000 h^{-1} \mathrm{Mpc}\right)$ and a lower resolution $\left(512^{3}\right.$ particles); hence we only perform the comparison 

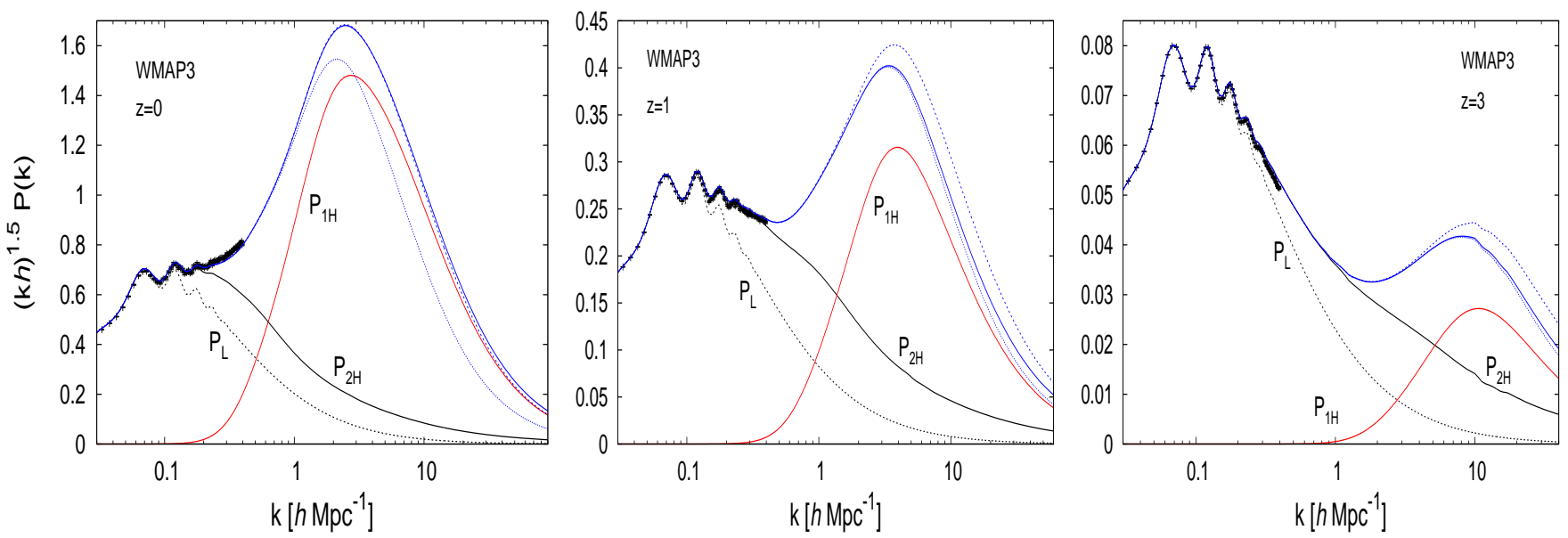

FIG. 16: Matter density power spectrum $P(k)$ at $z=0,1$, and 3 , from left to right, multiplied by a factor $(k h)^{1.5}$ as in Fig. 8 but for a different cosmology (WMAP3). Again, in addition to the result obtained with the mass-concentration relation (55) (solid line) we also show the results obtained using the mass-concentration relations from [63] and [64] (dashed lines).

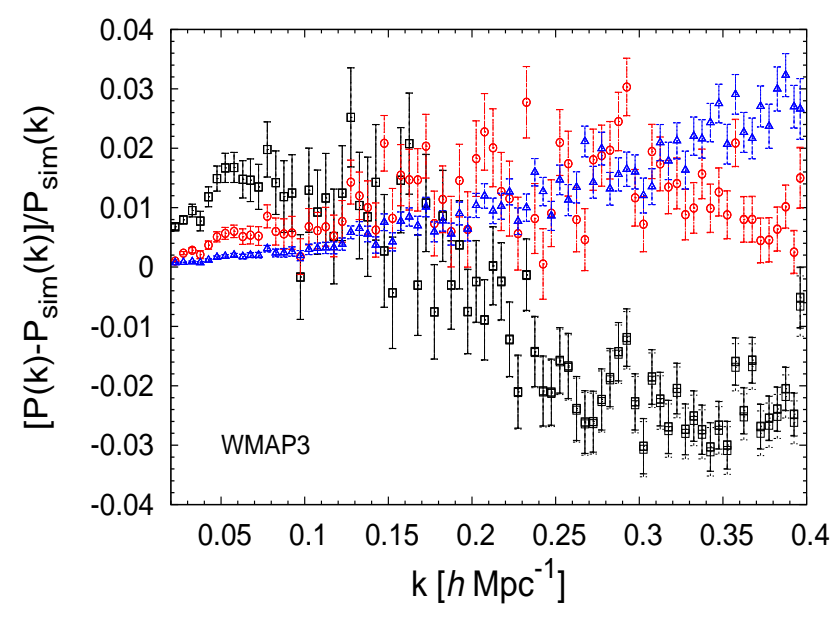

FIG. 17: Relative deviation between the density power spectrum predicted by our model and the result from numerical simulations, at $z=0$ (black squares), 1 (red circles), and 3 (blue triangles), as in Fig. 9 but for a different cosmology (WMAP3). We show the results (which almost coincide) obtained using the mass-concentration relation (55) and the ones from [63] and [64].

on large scales. On the other hand, they allow us to go down to redshift $z=0$.

We show our results for the power spectrum in Figs. 16 and [17. These results are similar to those obtained in Figs. 8 and 9 for the WMAP5 cosmology, with larger error bars in Fig. 17 because of the smaller box size of the simulations.

\section{Quintessence cosmology}

We finally consider a less standard cosmology, where the dark energy does not behave as a cosmological constant. Thus, we choose a dark energy equation-of-state parameter $w_{\mathrm{de}}=-1.281$, and $h=0.7737, \Omega_{\mathrm{m}}=0.23638$, $\sigma_{8}=0.7692$, and $n_{s}=1.0177$. We use the measurements from N-body simulations done in [14] for this cosmological model. These simulations are done in $2048 h^{-1} \mathrm{Mpc}$ boxes with $1024^{3}$ particles, and eight statistically independent realizations are available.

We show our results for the matter power spectrum in Figs. 18 and 19, and for the correlation function in Figs. 20 and 21]. We again obtain similar behaviors to those found for the LCDM WMAP5 cosmology in Sec. VA

This confirms that our method provides efficient predictions that can be used for a variety of cosmologies.

\section{CONCLUSION}

We have developed in this paper a new approach to a systematic modeling of the cosmological density field: instead of looking for explicit partial resummation schemes, we embed the standard perturbation theory within a realistic nonlinear ansatz. This automatically ensures a reasonable behavior on small scales, while being consistent with perturbative approaches up to the required order. Then, this allows us to obtain a reasonable matching with the highly nonlinear regime.

We have shown how this can be achieved within a Lagrangian-space framework. Because the lowestorder approximation that appears in this context is the Zel'dovich approximation [23], we first discussed the lack 

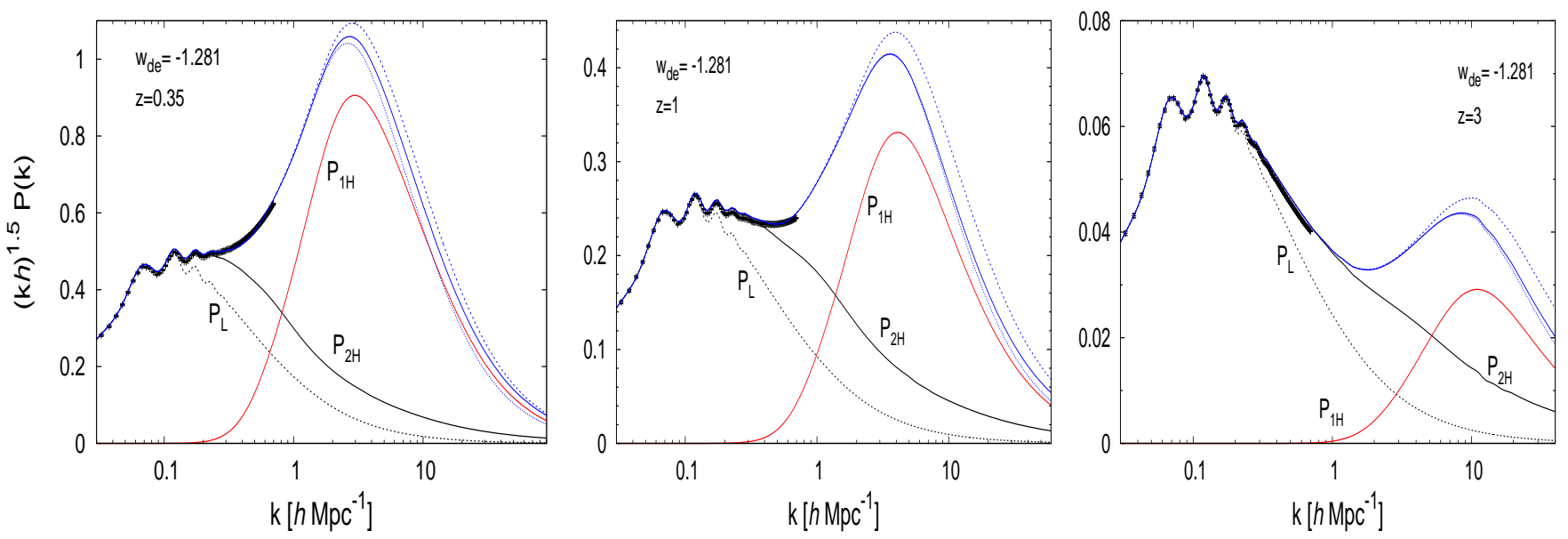

FIG. 18: Matter density power spectrum $P(k)$ at $z=0.35,1$, and 3 , from left to right, multiplied by a factor $(k h)^{1.5}$ as in Fig. 8 but for a different cosmology $\left(w_{\mathrm{de}}=-1.281\right)$. Again, in addition to the result obtained with the mass-concentration relation (55) (solid line) we also show the results obtained using the mass-concentration relations from [63] and 64] (dashed lines).

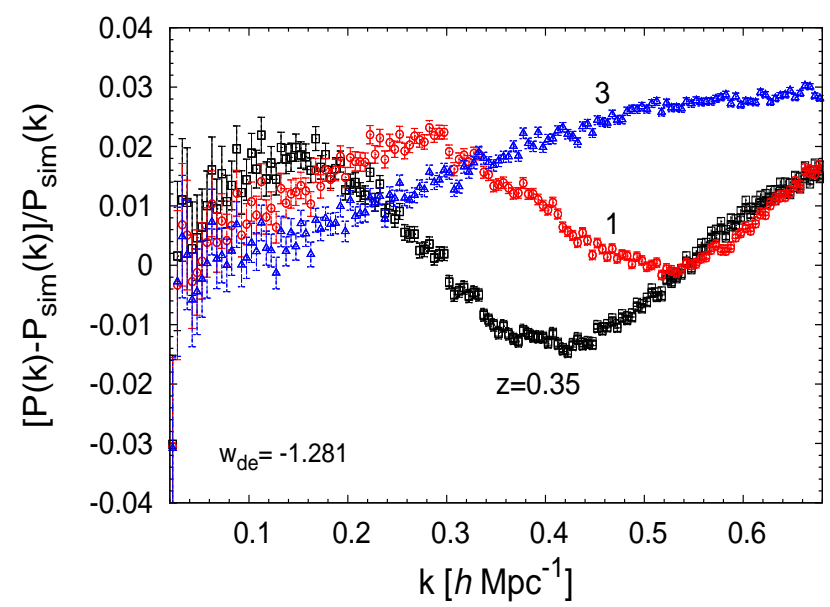

FIG. 19: Relative deviation between the density power spectrum predicted by our model and the result from numerical simulations, at $z=0.35$ (black squares), 1 (red circles), and 3 (blue triangles), as in Fig. 9 but for a different cosmology $\left(w_{\mathrm{de}}=-1.281\right)$. We show the results (which almost coincide) obtained using the mass-concentration relation (55) and the ones from [63] and 64].

of power on weakly nonlinear scales associated with this Gaussian approximation. Then, we explained how this can be partially cured by including higher-order terms (i.e., the skewness) while keeping a well-behaved ansatz. Because the Gaussian is the only probability distribution with a finite set of nonzero cumulants, this requires including nonzero cumulants at all orders and we describe a simple ansatz that provides a well-behaved probabil- ity distribution for the longitudinal displacement field. This provides a perturbative power spectrum that has the same qualitative properties as the true gravitational dynamics power spectrum. Moreover, it can be made consistent with the exact perturbative expansion up to the required order, while keeping all these qualitative properties. Here we only go up to order $P_{L}^{2}$, but higher orders could be taken into account by explicitly choosing the kurtosis and higher-order cumulants of the displacement field (at the price of a more complex ansatz for the cumulant-generating function).

Next, we argued that for a good description of intermediate scales it is important to include some effects associated with the shell-crossing regime. In particular, we pointed out that the small-scale behavior implied by the Zel'dovich power spectrum and most previous Lagrangian-space schemes, associated with the escape of particles to infinity, is not adequate, and a more realistic picture is provided by the adhesion model. We presented a simplified sticking model that captures some features of this trapping of particles within potential wells and describes the first stages of pancake formation. In particular, it captures the (nonperturbative) increase and change of sign of the skewness of the displacement field on small scales. This gives a "cosmic web" power spectrum that shows a broader high- $k$ tail than the original Zel'dovich power spectrum, with a universal $k^{-2}$ decay instead of the steeper-than- $k^{-3}$ falloff. The final expression for this "cosmic web" power spectrum is summarized 

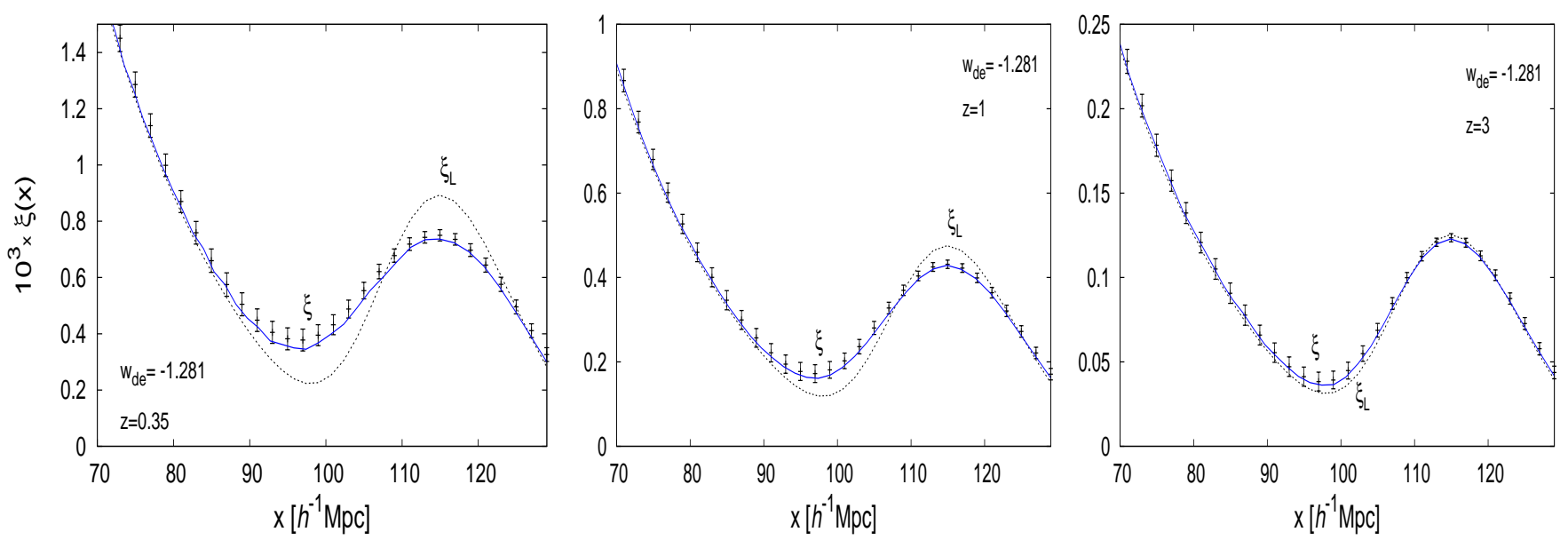

FIG. 20: Matter density correlation function $\xi(x)$ at $z=0.35,1$, and 3 , from left to right, multiplied by a factor $10^{3}$, as in Fig. 20 but for a different cosmology $\left(w_{\mathrm{de}}=-1.281\right)$.

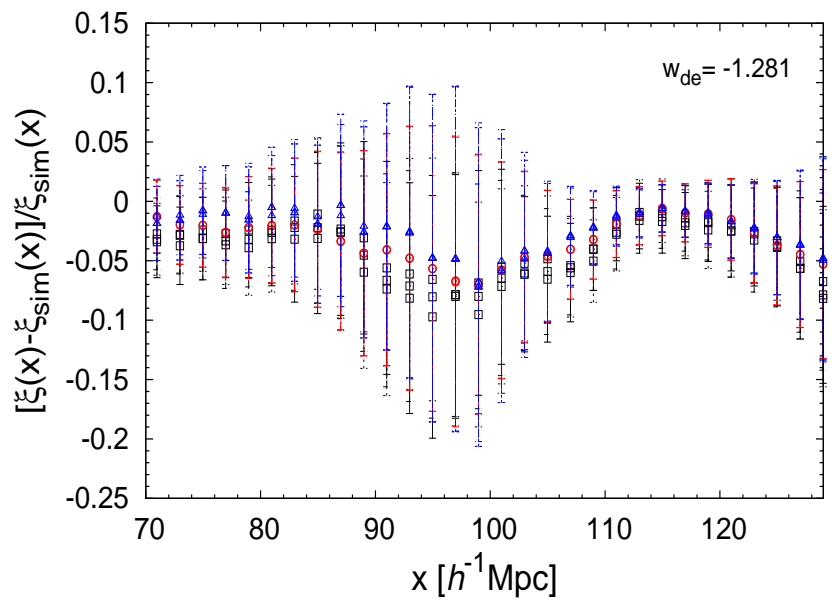

FIG. 21: Relative deviation between the two-point correlation $\xi(x)$ predicted by our model and the result from numerical simulations, at $z=0.35$ (squares), 1 (circles), and 3 (triangles), as in Fig. 12 but for a different cosmology $\left(w_{\mathrm{de}}=-1.281\right)$. We show the results (which almost coincide) obtained using the mass-concentration relation (55) and the ones from 63] and 64]. The error bars are the statistical error bars of the N-body simulations.

as follows [see Eq. (42)]:

$$
\begin{aligned}
& P_{\text {c.w. }}(k)=\int \frac{\mathrm{d} \Delta \mathbf{q}}{(2 \pi)^{3}} \frac{1}{1+A_{1}}\left\{e^{-\varphi_{\|}\left(-\mathrm{i} k \mu \Delta q \sigma_{\kappa_{\|}}^{2}\right) / \sigma_{\kappa_{\|}}^{2}}+A_{1}\right. \\
& \left.+\int_{0^{+}-\mathrm{i} \infty}^{0^{+}+\mathrm{i} \infty} \frac{\mathrm{d} y}{2 \pi \mathrm{i}} e^{-\varphi_{\|}(y) / \sigma_{\kappa_{\|}}^{2}}\left[\frac{1}{y}-\frac{1}{y+\mathrm{i} k \mu \Delta q \sigma_{\kappa_{\|}}^{2}}\right]\right\} \\
& \times e^{-\frac{1}{2} k^{2}\left(1-\mu^{2}\right) \sigma_{\perp}^{2}}
\end{aligned}
$$

with the quantities $\sigma_{\perp}^{2}, \sigma_{\kappa_{\|}}^{2}=\sigma_{\|}^{2} /(\Delta q)^{2}$ and $A_{1}$ re- spectively given by Eqs. (14), (15), and (40). For the cumulant-generating function $\varphi_{\|}$, we adopt the simple ansatz of Eq. (31), which is parameterized by the effective skewness (27) through the scale-dependent parameter $\alpha(\Delta q)$ [Eq. (32)].

This provides a well-behaved cosmic web power spectrum that can be combined with a halo model to describe all regimes of the cosmological density field, from large linear to small, highly nonlinear scales. The explicit expression for the power spectrum, given as the sum of the two contributions $P_{1 \mathrm{H}}(k)$ and $P_{2 \mathrm{H}}(k)$, is described in Sec. IV] [see Eqs. (49) and (52)].

A comparison with numerical simulations shows that our new proposition extends the range of validity of theoretical predictions over previous models. We obtain an accuracy better than $2 \%$ for $k \leq 0.3 h \mathrm{Mpc}^{-1}$ and better than $5 \%$ for $k \leq 3 h \mathrm{Mpc}^{-1}$, at $z \geq 0.35$. This also yields an accuracy of $2 \%$ on BAO scales for the two-point correlation function and of $5 \%$ down to $0.5 h^{-1} \mathrm{Mpc}$. We checked that a similar accuracy is obtained for an alternative less standard cosmology, with a dark energy equation-of-state parameter $w_{\mathrm{de}}=-1.28$.

Our approach should be generalized in two directions to provide a more direct comparison with observations. First, we should take into account redshift-space distortions, which should be possible within our Lagrangianspace framework. Second, we could consider the statistics of biased tracers, such as galaxies of different mass or luminosity. We leave these generalizations to further works.

The model developed in this paper can also be directly applied to several topics. First, following [73, 74], we can use our predictions for the 3D density field power spectrum to obtain the power spectrum of the weak-lensing shear (in the Born approximation), by integrating along the line of sight. Second, as in [75, 76], we can study less 


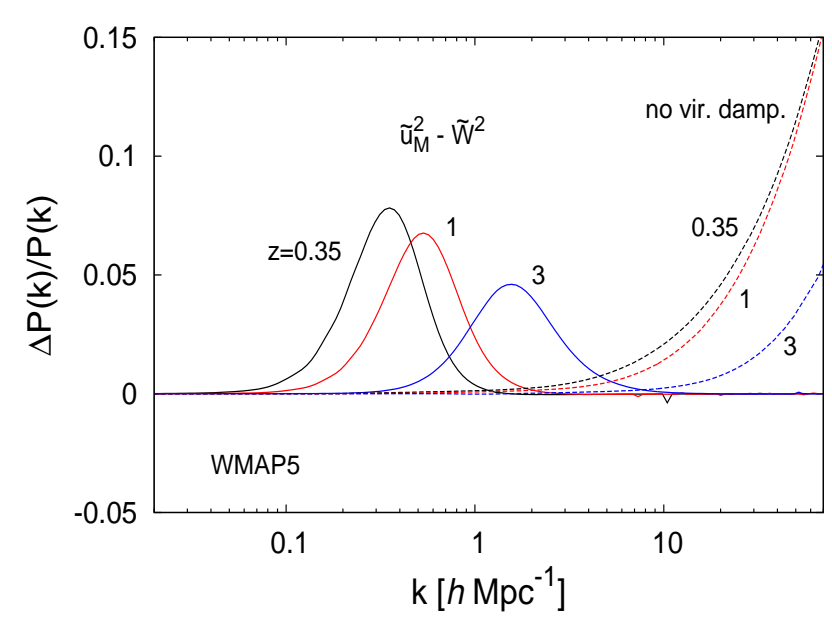

FIG. 22: Relative deviation from our model (49)-(52) of the power spectra obtained with a factor $\left(\tilde{u}_{M}^{2}-\tilde{W}^{2}\right)$ instead of $\left(\tilde{u}_{M}-\tilde{W}\right)^{2}$ in Eq.(49)) (left solid lines), or with a virial damping factor $\left\langle e^{\mathrm{ik} \cdot \Delta \mathbf{x}}\right\rangle_{\Delta q}^{\text {vir }}$ set to unity instead of Eq. (154) in Eq. (52) (right dashed lines). We show our results for the WMAP5 cosmology of Sec. $\mathrm{VA}$ at redshifts $z=0.35,1$, and 3 .

standard scenarios that involve modifications of gravity on cosmological scales, or consider the possible impact on the power spectrum of a warm dark matter component [77]. It should also be possible to study the effect of neutrinos [78] or of baryons [12] on the power spectrum.

\section{Acknowledgments}

This work has benefited from exchange visits supported by a bilateral grant from the Ministère des Affaires Etrangères et Européennes in France and the Japan Society for the Promotion of Science (JSPS). P. V. is supported in part by the French Agence Nationale de la Recherche under Grant ANR-12-BS05-0002. A.T. is supported in part by a Grant-in-Aid for Scientific Research from the JSPS (No. 24540257). T. N. is supported by a Grant-in-Aid for JSPS Fellows (PD: 22-181) and by the World Premier International Research Center Initiative (WPI Initiative), MEXT, Japan. Numerical computations for the present work have been carried out in part on Cray XT4 at the Center for Computational Astrophysics, CfCA, of the National Astronomical Observatory of Japan, and in part under the Interdisciplinary Computational Science Program in the Center for Computational Sciences, University of Tsukuba.

\section{Appendix A: Halo model details}

We show in Fig. 22 the impact on the matter power spectrum of some details of the halo model.

The solid curves show the relative deviation from our prediction that would be obtained by using a factor $\left(\tilde{u}_{M}^{2}-\tilde{W}^{2}\right)$, as in [11], instead of $\left(\tilde{u}_{M}-\tilde{W}\right)^{2}$ in Eq.(49). This only gives a $k^{2}$ decay at low $k$ instead of a $k^{4}$ tail (i.e., it satisfies matter conservation but not momentum conservation [47]). This would slightly overestimate the power spectrum on weakly nonlinear scales, where the one-halo term starts being non-negligible while remaining sensitive to its asymptotic low- $k$ behavior.

The dashed curves show the relative deviation from our prediction that would be obtained by neglecting the virial damping factor $\left\langle e^{\mathrm{ik} \cdot \Delta \mathbf{x}}\right\rangle_{\Delta q}^{\mathrm{vir}}$ in Eq.(52). This would overestimate the power spectrum in the highly nonlinear regime on small scales, which are sensitive to motions within halo cores. However, on these scales our model suffers from other uncertainties, due to the limited accuracy of halo profiles and concentration parameters, and to the approximate form of our two-halo contribution.
[1] F. Bernardeau, S. Colombi, E. Gaztañaga, and R. Scoccimarro, Phys. Rep. 367, 1 (2002), arXiv:astro$\mathrm{ph} / 0112551$.

[2] P. Valageas, Astr. \& Astrophys. 476, 31 (2007), 0706.2593.

[3] F. Bernardeau and P. Valageas, Phys. Rev. D 78, 083503 (2008), 0805.0805.

[4] F. Bernardeau and P. Valageas, Phys. Rev. D 85, 023516 (2012), 1109.4223.

[5] M. Crocce and R. Scoccimarro, Phys. Rev. D 73, 063519 (2006), arXiv:astro-ph/0509418.

[6] M. Crocce and R. Scoccimarro, Phys. Rev. D 73, 063520 (2006), arXiv:astro-ph/0509419.

[7] P. Valageas, Astr. \& Astrophys. 465, 725 (2007), arXiv:astro-ph/0611849.

[8] A. Taruya and T. Hiramatsu, Astrophys. J. 674, 617 (2008), 0708.1367.
[9] M. Pietroni, JCAP 10, 36 (2008), 0806.0971.

[10] F. Bernardeau, M. Crocce, and R. Scoccimarro, Phys. Rev. D 78, 103521 (2008), 0806.2334.

[11] P. Valageas and T. Nishimichi, Astr. \& Astrophys. 527, A $87+$ (2011), 1009.0597.

[12] F. Bernardeau, N. Van de Rijt, and F. Vernizzi, Phys. Rev. D 87, 043530 (2013), 1209.3662.

[13] S. Anselmi and M. Pietroni, JCAP 12, 013 (2012), 1205.2235

[14] A. Taruya, F. Bernardeau, T. Nishimichi, and S. Codis, Phys. Rev. D 86, 103528 (2012), 1208.1191.

[15] P. Valageas and T. Nishimichi, Astr. $E$ Astrophys. 532, A4+ (2011), 1102.0641.

[16] T. Matsubara, Phys. Rev. D 77, 063530 (2008), 0711.2521.

[17] T. Okamura, A. Taruya, and T. Matsubara, JCAP 8, 012 (2011), 1105.1491. 
[18] S. N. Gurbatov, A. I. Saichev, and S. F. Shandarin, Mon. Not. R. Astr. Soc. 236, 385 (1989).

[19] P. Valageas, Astr. \& Astrophys. 526, A67+ (2011), 1009.0106.

[20] E. Komatsu, J. Dunkley, M. R. Nolta, C. L. Bennett, B. Gold, G. Hinshaw, N. Jarosik, D. Larson, M. Limon, L. Page, et al., Astrophys. J. Suppl. Ser. 180, 330 (2009), 0803.0547.

[21] P. Schneider and M. Bartelmann, Mon. Not. R. Astr. Soc. 273, 475 (1995).

[22] A. N. Taylor and A. J. S. Hamilton, Mon. Not. R. Astr. Soc. 282, 767 (1996), arXiv:astro-ph/9604020.

[23] Y. B. Zel'Dovich, Astr. ES Astrophys. 5, 84 (1970).

[24] P. Valageas, Astr. \& Astrophys. 484, 79 (2008), 0711.3407.

[25] T. Buchert, A. L. Melott, and A. G. Weiss, Astr. Es Astrophys. 288, 349 (1994), arXiv:astro-ph/9309056.

[26] F. R. Bouchet, S. Colombi, E. Hivon, and R. Juszkiewicz, Astr. $\&$ Astrophys. 296, 575 (1995), arXiv:astro$\mathrm{ph} / 9406013$.

[27] R. Scoccimarro, Mon. Not. R. Astr. Soc. 299, 1097 (1998), arXiv:astro-ph/9711187.

[28] S. Matarrese and M. Pietroni, JCAP 6, 26 (2007), arXiv:astro-ph/0703563.

[29] J. Carlson, B. Reid, and M. White, ArXiv e-prints (2012), 1209.0780.

[30] F. Bernardeau and P. Valageas, Phys. Rev. D 81, 043516 (2010), 0912.0356.

[31] M. Vergassola, B. Dubrulle, U. Frisch, and A. Noullez, Astr. \& Astrophys. 289, 325 (1994).

[32] F. Bernardeau and P. Valageas, Phys. Rev. E 82, 016311 (2010), 0912.3603.

[33] P. Valageas and F. Bernardeau, Phys. Rev. D 83, 043508 (2011), 1009.1974.

[34] I. A. Bogaevsky, Sbornik: Mathematics 197 (2006), math-ph/0407073, URL http://arxiv.org/abs/math-ph/0407073.

[35] P. Coles, A. L. Melott, and S. F. Shandarin, Mon. Not. R. Astr. Soc. 260, 765 (1993).

[36] B. S. Sathyaprakash, V. Sahni, D. Munshi, D. Pogosyan, and A. L. Melott, Mon. Not. R. Astr. Soc. 275, 463 (1995), arXiv:astro-ph/9408089.

[37] R. Balian and R. Schaeffer, Astr. E Astrophys. 220, 1 (1989).

[38] R. Balian and R. Schaeffer, Astr. \& Astrophys. 226, 373 (1989).

[39] P. J. E. Peebles, The large-scale structure of the universe (Princeton University Press, Princeton, N.J., USA, 1980).

[40] A. Noullez and M. Vergassola, J. Sci. Comput. 9, 259 (1994).

[41] Y. Brenier, U. Frisch, M. Hénon, G. Loeper, S. Matarrese, R. Mohayaee, and A. Sobolevskiü, Mon. Not. R. Astr. Soc. 346, 501 (2003), arXiv:astro-ph/0304214.

[42] T. Buchert, Mon. Not. R. Astr. Soc. 267, 811 (1994), arXiv:astro-ph/9309055.

[43] A. Cooray and R. Sheth, Phys. Rep. 372, 1 (2002), astro$\mathrm{ph} / 0206508$.

[44] P. Valageas, Astr. \& Astrophys. 508, 93 (2009), 0905.2277 .

[45] P. Valageas, Astr. \& Astrophys. 382, 450 (2002), arXiv:astro-ph/0107333.

[46] J. F. Navarro, C. S. Frenk, and S. D. M. White, Astrophys. J. 490, 493 (1997), arXiv:astro-ph/9611107.
[47] P. J. E. Peebles, Astr. \& Astrophys. 32, 391 (1974).

[48] M. Pietroni, G. Mangano, N. Saviano, and M. Viel, JCAP 1, 019 (2012), 1108.5203.

[49] J. J. M. Carrasco, M. P. Hertzberg, and L. Senatore, Journal of High Energy Physics 9, 82 (2012), 1206.2926.

[50] J. Burgers, The nonlinear diffusion equation. Asymptotic solutions and statistical problems. (Dordrecht - Boston: D. Reidel Publishing Company. X, 173 p., 1974).

[51] J. Bertoin, Commun. Math. Phys. 193, 397 (1998).

[52] P. Valageas, Journal of Statistical Physics 134, 589 (2009), 0810.4332.

[53] L. Frachebourg and P. A. Martin, Journal of Fluid Mechanics 417, 323 (2000), arXiv:cond-mat/9905056.

[54] P. Valageas, Journal of Statistical Physics 137, 729 (2009), 0903.0956.

[55] A. Domínguez, Phys. Rev. D 62, 103501 (2000), arXiv:astro-ph/0009414.

[56] T. Buchert and A. Domínguez, Astr. $E$ Astrophys. 438, 443 (2005), arXiv:astro-ph/0502318.

[57] P. Valageas, Astr. \& Astrophys. 379, 8 (2001), arXiv:astro-ph/0107015.

[58] P. Valageas, Astr. \&6 Astrophys. 421, 23 (2004), arXiv:astro-ph/0307008.

[59] S. V. Tassev, JCAP 10, 022 (2011), 1012.0282.

[60] D. J. Eisenstein, I. Zehavi, D. W. Hogg, R. Scoccimarro, M. R. Blanton, R. C. Nichol, R. Scranton, H.-J. Seo, M. Tegmark, Z. Zheng, et al., Astrophys. J. 633, 560 (2005), arXiv:astro-ph/0501171.

[61] E. A. Kazin, M. R. Blanton, R. Scoccimarro, C. K. McBride, A. A. Berlind, N. A. Bahcall, J. Brinkmann, P. Czarapata, J. A. Frieman, S. M. Kent, et al., Astrophys. J. 710, 1444 (2010), 0908.2598.

[62] L. Anderson, E. Aubourg, S. Bailey, F. Beutler, A. S. Bolton, J. Brinkmann, J. R. Brownstein, C.-H. Chuang, A. J. Cuesta, K. S. Dawson, et al., ArXiv e-prints (2013), 1303.4666 .

[63] A. R. Duffy, J. Schaye, S. T. Kay, and C. Dalla Vecchia, Mon. Not. R. Astr. Soc. 390, L64 (2008), 0804.2486.

[64] S. Bhattacharya, S. Habib, K. Heitmann, and A. Vikhlinin, Astrophys. J. 766, 32 (2013), 1112.5479.

[65] M. Crocce and R. Scoccimarro, Phys. Rev. D 77, 023533 (2008), 0704.2783.

[66] A. Taruya, T. Nishimichi, S. Saito, and T. Hiramatsu, Phys. Rev. D 80, 123503 (2009), 0906.0507.

[67] T. Guillet, R. Teyssier, and S. Colombi, Mon. Not. R. Astr. Soc. 405, 525 (2010), 0905.2615.

[68] M. P. van Daalen, J. Schaye, C. M. Booth, and C. Dalla Vecchia, Mon. Not. R. Astr. Soc. 415, 3649 (2011), 1104.1174

[69] E. Semboloni, H. Hoekstra, J. Schaye, M. P. van Daalen, and I. G. McCarthy, Mon. Not. R. Astr. Soc. 417, 2020 (2011), 1105.1075.

[70] D. J. Eisenstein, H.-J. Seo, and M. White, Astrophys. J. 664, 660 (2007), arXiv:astro-ph/0604361.

[71] D. J. Eisenstein, H.-J. Seo, E. Sirko, and D. N. Spergel, Astrophys. J. 664, 675 (2007), arXiv:astro-ph/0604362.

[72] T. Nishimichi, A. Shirata, A. Taruya, K. Yahata, S. Saito, Y. Suto, R. Takahashi, N. Yoshida, T. Matsubara, N. Sugiyama, et al., Publ. Astron. Soc. Japan 61, 321 (2009), 0810.0813.

[73] P. Valageas, M. Sato, and T. Nishimichi, Astr. 86 Astrophys. 541, A161 (2012), 1111.7156.

[74] P. Valageas, M. Sato, and T. Nishimichi, Astr. ES Astrophys. 541, A162 (2012), 1112.1495. 
[75] K. Koyama, A. Taruya, and T. Hiramatsu, Phys. Rev. D 79, 123512 (2009), 0902.0618.

[76] P. Brax and P. Valageas, Phys. Rev. D 86, 063512 (2012), 1205.6583.
[77] P. Valageas, Phys. Rev. D 86, 123501 (2012), 1206.0554.

[78] J. Lesgourgues, S. Matarrese, M. Pietroni, and A. Riotto, JCAP 6, 17 (2009), 0901.4550. 